\title{
Normal forms of Levi-flat hypersurfaces with Arnold type singularities
}

\author{
ARTURO FERnÁNDEZ-PÉREZ
}

\begin{abstract}
In this paper we study normal forms of Levi-flat hypersurfaces with singularities. We prove a result analogous to the Burns-Gong theorem for the existence of rigid normal forms of Levi-flat hypersurfaces which are defined by the vanishing of the real part of $A_{k}, D_{k}, E_{k}$ singularities.
\end{abstract}

Mathematics Subject Classification (2010): 32V40 (primary); 32S65 (secondary).

\section{Introduction}

In 1999 D. Burns and X. Gong proved the following result ( $c f$. [5]):

Theorem 1.1 (Burns-Gong). Let $M$ be a germ of real analytic Levi-flat hypersurface at $0 \in \mathbb{C}^{n}$, with $n \geq 2$, defined by

$$
\mathcal{R} e\left(z_{1}^{2}+\ldots+z_{n}^{2}\right)+H(z, \bar{z})=0
$$

with $H(z, \bar{z})=O\left(|z|^{3}\right)$, and $H(z, \bar{z})=\bar{H}(\bar{z}, z)$. Then there exists a holomorphic coordinate system such that

$$
M=\left(\mathcal{R} e\left(x_{1}^{2}+\ldots+x_{n}^{2}\right)=0\right) .
$$

This result can be viewed as a Morse Lemma for Levi-flat hypersurfaces and it is a normal form in the case of a generic (Morse) singularity. Singular Levi-flat hypersurfaces have been studied by many authors, see for example Bedford [4], Brunella [6], Cerveau-Lins Neto [8], Lebl [16] and the author [12,13]. In the same spirit the purpose of this paper is to prove the existence of normal forms of Levi-flat hypersurfaces with Arnold type singularities. More precisely, we are interested in

This work was partially supported by FAPEMIG - Brasil and PRPq - Universidade Federal de Minas Gerais, UFMG. Finally, I would like to thank the referee for his comments and suggestions. Received December 6, 2011; accepted in revised form August 9, 2012. 
obtaining normal forms of Levi-flat hypersurfaces which are defined by the vanishing of the real part of $A_{k}, D_{k}, E_{k}$ singularities.

One motivation for considering $A_{k}, D_{k}, E_{k}$ singularities is the following: when we consider the problem of classifying holomorphic germs $f$ with an isolated singularity at $0 \in \mathbb{C}^{n}$, with respect to holomorphic changes of coordinates, the list starts with the famous $A_{k}, D_{k}, E_{k}$ singularities or simple singularities, see for instance Arnold's papers [1,2]:

Table 1.1. $A_{k}, D_{k}, E_{k}$ singularities.

\begin{tabular}{clc}
\hline Type & Normal form & Conditions \\
\hline$A_{k}$ & $z_{1}^{2}+z_{2}^{k+1}+\ldots+z_{n}^{2}$, & $k \geq 1$ \\
$D_{k}$ & $z_{1}^{2} z_{2}+z_{2}^{k-1}+z_{3}^{2}+\ldots+z_{n}^{2}$, & $k \geq 4$ \\
$E_{6}$ & $z_{1}^{4}+z_{2}^{3}+z_{3}^{2}+\ldots+z_{n}^{2}$ & \\
$E_{7}$ & $z_{1}^{3} z_{2}+z_{2}^{3}+z_{3}^{2}+\ldots+z_{n}^{2}$ & \\
$E_{8}$ & $z_{1}^{5}+z_{2}^{3}+z_{3}^{2}+\ldots+z_{n}^{2}$ & \\
\hline
\end{tabular}

Several characterizations of the $A_{k}, D_{k}, E_{k}$ singularities are well-known, see for instance [10]. Let us give the precise statement of these results. Let $M$ be a germ at $0 \in \mathbb{C}^{n}$ of an irreducible real analytic hypersurface defined by $(F=0)$. The singular set of $M$ is defined by $\operatorname{Sing}(M)=(F=0) \cap(d F=0)$ and its smooth part $(F=0) \backslash(d F=0)$ will be denoted by $M^{*}$. The Levi distribution $L$ on $M^{*}$ is defined by

$$
L_{p}:=\operatorname{Ker}(\partial F(p)) \subset T_{p} M^{*}=\operatorname{Ker}(d F(p)), \text { for any } p \in M^{*} .
$$

We shall say that $M$ is Levi-flat if the Levi distribution $L$ on $M^{*}$ is integrable. The integrability condition of $L$ implies that $M$ is smoothly foliated by immersed complex manifolds of complex dimension $n-1$. The Levi foliation, that we denote by $\mathcal{L}$, is the foliation defined by this distribution.

The Levi distribution $L$ on $M^{*}$ can be defined by the real analytic 1-form $\eta=\left.i(\partial F-\bar{\partial} F)\right|_{M^{*}}$, which will be called the Levi 1-form of $F$. The integrability condition is equivalent to $\left.(\partial F-\bar{\partial} F) \wedge \partial \bar{\partial} F\right|_{M^{*}}=0$. Since $d F=\partial F+\bar{\partial} F$, this is also equivalent to

$$
\partial F(p) \wedge \bar{\partial} F(p) \wedge \partial \bar{\partial} F(p)=0, \quad \forall p \in M .
$$

See the book [3] for the basic language and background about Levi-flat hypersurfaces. Before stating our result, let us describe some known results and examples.

Example 1.2. If $M$ is smooth, by a classical result of E. Cartan there exists a holomorphic coordinate system $\left(z_{1}, \ldots, z_{n}\right) \in \mathbb{C}^{n}$ such that $M$ can be represented as $M=\left(\mathcal{R e}\left(z_{n}\right)=0\right)$.

Example 1.3. If $h:\left(\mathbb{C}^{n}, 0\right) \rightarrow(\mathbb{C}, 0)$ is holomorphic and non-constant then the analytic set defined by $M=(\mathcal{R} e(h)=0)$ is Levi-flat and $\operatorname{Sing}(M)=\operatorname{crit}(f) \cap M$, 
where $\operatorname{crit}(f)$ is the set of critical points of $f$. The leaves of $\mathcal{L}$ on $M$ are the imaginary levels of $h$.

Example 1.4. Let $M=\left(\mathcal{R} e\left(z_{1}^{2}+\ldots+z_{n}^{2}\right)+H(z, \bar{z})=0\right)$ be as in Theorem 1.1 then there exists a holomorphic coordinate system such that $M=\left(\mathcal{R} e\left(x_{1}^{2}+\ldots+\right.\right.$ $\left.x_{n}^{2}\right)=0$ ), we remark that it is a normal form (Levi-flat) of $A_{1}$ type. This result was generalized in [12], where we considered the real part of a homogeneous polynomial of degree $k \geq 2$ with an isolated singularity.

Example 1.5. Let $M$ be a germ of real analytic Levi-flat hypersurface at $0 \in \mathbb{C}^{2}$ defined by $F=0$, where

$$
F(x, y)=\operatorname{Re}\left(x^{2} y+y^{3}\right)+H(x, y, \bar{x}, \bar{y})
$$

with $H(x, y, \bar{x}, \bar{y})=O\left(|(x, y)|^{4}\right)$ and $H(x, y, \bar{x}, \bar{y})=\bar{H}(\bar{x}, \bar{y}, x, y)$. Then in [12] we proved that there exists a holomorphic coordinate system such that

$$
M=\left(\mathcal{R} e\left(x_{1}^{2} y_{1}+y_{1}^{3}\right)=0\right),
$$

which is a normal form of $D_{4}$ type when $n=2$. On the other hand, if $n \geq 3$ the analogous result is also valid by [12, Theorem 2].

These results were proved using techniques of holomorphic foliations developed in [11]. This new approach provides new normal forms of Levi-flat hypersurfaces. Our main result is an Arnold type result for singular Levi-flat hypersurfaces.

Theorem 1. Let $M=F^{-1}(0)$ be a germ at $0 \in \mathbb{C}^{n}$, with $n \geq 2$, of irreducible real analytic Levi-flat hypersurface. Suppose that $F$ is of one of the following types:

(a) $F(z)=\mathcal{R} e\left(z_{1}^{2}+z_{2}^{k+1}+z_{3}^{2}+\ldots+z_{n}^{2}\right)+H(z, \bar{z})$, where $k \geq 2$ and

$$
H(z, \bar{z})=O\left(|z|^{k+2}\right), H(z, \bar{z})=\bar{H}(\bar{z}, z) .
$$

(b) $F(z)=\operatorname{Re}\left(z_{1}^{2} z_{2}+z_{2}^{k-1}+z_{3}^{2}+\ldots+z_{n}^{2}\right)+H(z, \bar{z})$, where $k \geq 5$ and

$$
H(z, \bar{z})=O\left(|z|^{k}\right), H(z, \bar{z})=\bar{H}(\bar{z}, z) .
$$

(c) $F(z)=\mathcal{R} e\left(z_{1}^{4}+z_{2}^{3}+z_{3}^{2}+\ldots+z_{n}^{2}\right)+H(z, \bar{z})$, where

$$
H(z, \bar{z})=O\left(|z|^{5}\right), H(z, \bar{z})=\bar{H}(\bar{z}, z) .
$$

(d) $F(z)=\mathcal{R} e\left(z_{1}^{3} z_{2}+z_{2}^{3}+z_{3}^{2}+\ldots+z_{n}^{2}\right)+H(z, \bar{z})$, where

$$
H(z, \bar{z})=O\left(|z|^{5}\right), H(z, \bar{z})=\bar{H}(\bar{z}, z) .
$$

(e) $F(z)=\mathcal{R} e\left(z_{1}^{5}+z_{2}^{3}+z_{3}^{2}+\ldots+z_{n}^{2}\right)+H(z, \bar{z})$, where

$$
H(z, \bar{z})=O\left(|z|^{6}\right), H(z, \bar{z})=\bar{H}(\bar{z}, z) .
$$


Then there exists a germ of biholomorphism $\varphi:\left(\mathbb{C}^{n}, 0\right) \rightarrow\left(\mathbb{C}^{n}, 0\right)$ such that

(a) $\varphi(M)=\left(\mathcal{R} e\left(z_{1}^{2}+z_{2}^{k+1}+z_{3}^{2}+\ldots+z_{n}^{2}\right)=0\right)$,

(b) $\varphi(M)=\left(\mathcal{R e} e\left(z_{1}^{2} z_{2}+z_{2}^{k-1}+z_{3}^{2}+\ldots+z_{n}^{2}\right)=0\right)$,

(c) $\varphi(M)=\left(\mathcal{R} e\left(z_{1}^{4}+z_{2}^{3}+z_{3}^{2}+\ldots+z_{n}^{2}\right)=0\right)$,

(d) $\varphi(M)=\left(\mathcal{R e}\left(z_{1}^{3} z_{2}+z_{2}^{3}+z_{3}^{2}+\ldots+z_{n}^{2}\right)=0\right)$,

(e) $\varphi(M)=\left(\mathcal{R e}\left(z_{1}^{5}+z_{2}^{3}+z_{3}^{2}+\ldots+z_{n}^{2}\right)=0\right)$, respectively.

We find the following list:

Table 1.2. Levi-flat hypersurfaces with $A_{k}, D_{k}, E_{k}$ singularities.

\begin{tabular}{clc}
\hline Type & Normal form & Conditions \\
\hline$A_{k}$ & $\mathcal{R} e\left(z_{1}^{2}+z_{2}^{k+1}+\ldots+z_{n}^{2}\right)=0$ & $k \geq 1$ \\
$D_{k}$ & $\mathcal{R e}\left(z_{1}^{2} z_{2}+z_{2}^{k-1}+z_{3}^{2}+\ldots+z_{n}^{2}\right)=0$ & $k \geq 4$ \\
$E_{6}$ & $\mathcal{R e}\left(z_{1}^{4}+z_{2}^{3}+z_{3}^{2}+\ldots+z_{n}^{2}\right)=0$ & \\
$E_{7}$ & $\mathcal{R e}\left(z_{1}^{3} z_{2}+z_{2}^{3}+z_{3}^{2}+\ldots+z_{n}^{2}\right)=0$ & \\
$E_{8}$ & $\mathcal{R e}\left(z_{1}^{5}+z_{2}^{3}+z_{3}^{2}+\ldots+z_{n}^{2}\right)=0$ & \\
\hline
\end{tabular}

The main tool for proving this theorem is a result of Cerveau and Lins Neto [8], that gives sufficient conditions for a Levi-flat hypersurface to be defined by the zeros of the real part of a holomorphic function.

This paper is organized as follows: in Section 2, we recall some properties and known results about singular Levi-flat hypersurfaces. Section 3 is devoted to recall the notions of weighted projective space and weighted blow-ups. In Section 4 we prove Theorem 1 for dimension $n \geq 3$. Finally, in Section 5 we conclude the proof for dimension two.

ACKNOWLEDGEMENTS. I wish to thank my advisor Alcides Lins Neto for many useful discussions and many suggestions for improvements of these results. I also would like to thank Hossein Movasati, Jorge Vitório Pereira and Daniel Panazzolo for helpful conversations about this work.

\section{Preliminaries}

Let us fix some notation that will be used from now on:

1. $\mathcal{O}_{n}$ : the ring of germs of holomorphic functions at $0 \in \mathbb{C}^{n}$; $\mathcal{O}(U)$ : the set of holomorphic functions on the open set $U \subset \mathbb{C}^{n}$; 
2. $\mathcal{O}_{n}^{*}=\left\{f \in \mathcal{O}_{n} \mid f(0) \neq 0\right\}$, $\mathcal{O}^{*}(U)=\{f \in \mathcal{O}(U) \mid f(z) \neq 0, \forall z \in U\}$

3. $\mathcal{M}_{n}=\left\{f \in \mathcal{O}_{n} \mid f(0)=0\right\}$ the maximal ideal of $\mathcal{O}_{n}$;

4. $\mathcal{A}_{n}$ : the ring of germs at $0 \in \mathbb{C}^{n}$ of complex valued real analytic functions;

5. $\mathcal{A}_{n \mathbb{R}}$ : the ring of germs at $0 \in \mathbb{C}^{n}$ of real valued real analytic functions. Note that $F \in \mathcal{A}_{n}$ is in $\mathcal{A}_{n \mathbb{R}}$ if and only if $F=\bar{F}$;

6. Diff( $\left(\mathbb{C}^{n}, 0\right)$ : the group of germs at $0 \in \mathbb{C}^{n}$ of holomorphic diffeomorphisms $f:\left(\mathbb{C}^{n}, 0\right) \rightarrow\left(\mathbb{C}^{n}, 0\right)$ with the operation of composition;

7. $j_{0}^{k}(f)$ : the $k$-jet at $0 \in \mathbb{C}^{n}$ of $f \in \mathcal{O}_{n}$.

Definition 2.1. Two germs $f, g \in \mathcal{O}_{n}$ are right-equivalent if there exists $\phi \in$ $\operatorname{Diff}\left(\mathbb{C}^{n}, 0\right)$ such that $f \circ \phi^{-1}=g$.

The local algebra of $f \in \mathcal{O}_{n}$ is by definition

$$
A_{f}:=\mathcal{O}_{n} /\left\langle\partial f / \partial z_{1}, \ldots, \partial f / \partial z_{n}\right\rangle \text {. }
$$

We denote by $\mu(f, 0):=\operatorname{dim} A_{f}$ the Milnor number of $f$ at $0 \in \mathbb{C}^{n}$. This number is finite if and only if 0 is an isolated singularity of $f$.

Definition 2.2. A germ $f \in \mathcal{O}_{n}$ is said to be quasihomogeneous of degree $d$ with indices $\alpha_{1}, \ldots, \alpha_{n}$ if for any $\lambda \in \mathbb{C}^{*}$ and $\left(z_{1}, \ldots, z_{n}\right) \in \mathbb{C}^{n}$ we have

$$
f\left(\lambda^{\alpha_{1}} z_{1}, \ldots, \lambda^{\alpha_{n}} z_{n}\right)=\lambda^{d} f\left(z_{1}, \ldots, z_{n}\right) .
$$

The index $\alpha_{s}$ is also called the weight of the variable $z_{s}$.

\subsection{Complexification of a Levi-flat hypersurface}

Given $F \in \mathcal{A}_{n}$, we can write its Taylor series at $0 \in \mathbb{C}^{n}$ as

$$
F(z)=\sum_{\mu, v} F_{\mu \nu} z^{\mu} \bar{z}^{\nu}
$$

where $F_{\mu v} \in \mathbb{C}, \mu=\left(\mu_{1}, \ldots, \mu_{n}\right), v=\left(v_{1}, \ldots, v_{n}\right), z^{\mu}=z_{1}^{\mu_{1}} \ldots z_{n}^{\mu_{n}}, \bar{z}^{v}=$ $\bar{z}_{1}^{v_{1}} \ldots \bar{z}_{n}^{v_{n}}$. When $F \in \mathcal{A}_{n \mathbb{R}}$, the coefficients $F_{\mu \nu}$ satisfy

$$
\bar{F}_{\mu \nu}=F_{\nu \mu}
$$

The complexification $F_{\mathbb{C}} \in \mathcal{O}_{2 n}$ of $F$ is defined by the series

$$
F_{\mathbb{C}}(z, w)=\sum_{\mu, \nu} F_{\mu \nu} z^{\mu} w^{\nu}
$$

If the series in (2.1) converges in the polydisk $D_{r}^{n}=\left\{z \in \mathbb{C}^{n}:\left|z_{j}\right|<r\right\}$ then the series in (2.2) converges in the polydisk $D_{r}^{2 n}$. Moreover, $F(z)=F_{\mathbb{C}}(z, \bar{z})$ for all $z \in D_{r}^{n}$. 
Let $M=F^{-1}(0)$ be a Levi-flat hypersurface, where $F \in \mathcal{A}_{n \mathbb{R}}$. The complexification $\eta_{\mathbb{C}}$ of its Levi 1-form $\eta=i(\partial F-\bar{\partial} F)$ can be written as

$$
\eta_{\mathbb{C}}=i\left(\partial_{z} F_{\mathbb{C}}-\partial_{w} F_{\mathbb{C}}\right)=i \sum_{\mu, \nu}\left(F_{\mu \nu} w^{\nu} d\left(z^{\mu}\right)-F_{\mu \nu} z^{\mu} d\left(w^{\nu}\right)\right) .
$$

The complexification $M_{\mathbb{C}}$ of $M$ is defined as $M_{\mathbb{C}}=F_{\mathbb{C}}^{-1}(0)$ and its smooth part is $M_{\mathbb{C}}^{*}=M_{\mathbb{C}} \backslash\left(d F_{\mathbb{C}}=0\right)$. Clearly $M_{\mathbb{C}}$ defines a complex subvariety of dimension $2 n-1$. The integrability condition of $\eta=\left.i(\partial F-\bar{\partial} F)\right|_{M^{*}}$ implies that $\left.\eta_{\mathbb{C}}\right|_{M_{\mathbb{C}}^{*}}$ is integrable. Therefore $\left.\eta_{\mathbb{C}}\right|_{M_{\mathbb{C}}^{*}}=0$ defines a holomorphic foliation $\mathcal{L}_{\mathbb{C}}$ on $M_{\mathbb{C}}^{*}$ that will be called the complexification of $\mathcal{L}$.

Remark 2.3. Let $\eta=i(\partial F-\bar{\partial} F)$ and $\eta_{\mathbb{C}}$ be as before. Then $\left.\eta\right|_{M^{*}}$ and $\left.\eta_{\mathbb{C}}\right|_{M_{\mathbb{C}}^{*}}$ define $\mathcal{L}$ and $\mathcal{L}_{\mathbb{C}}$, respectively. Set $\alpha=\sum_{j=1}^{n} \frac{\partial F_{\mathbb{C}}}{\partial z_{j}} d z_{j}$ and $\beta=\sum_{j=1}^{n} \frac{\partial F_{\mathbb{C}}}{\partial w_{j}} d w_{j}$. Hence $d F_{\mathbb{C}}=\alpha+\beta$ and $\eta_{\mathbb{C}}=i(\alpha-\beta)$, so that

$$
\left.\eta \mathbb{C}\right|_{M_{\mathbb{C}}^{*}}=\left.2 i \alpha\right|_{M_{\mathbb{C}}^{*}}=-\left.2 i \beta\right|_{M_{\mathbb{C}}^{*}}
$$

In particular, $\left.\alpha\right|_{M_{\mathbb{C}}^{*}}$ and $\left.\beta\right|_{M_{\mathbb{C}}^{*}}$ define $\mathcal{L}_{\mathbb{C}}$.

\subsection{Holomorphic foliations and Levi-flat hypersurfaces}

This section is devoted to recalling some results about Levi-flat hypersurfaces invariant by holomorphic foliations.

Definition 2.4. Let $\mathcal{F}$ and $M=F^{-1}(0)$ be germs at $\left(\mathbb{C}^{n}, 0\right)$, with $n \geq 2$, of a codimension-one singular holomorphic foliation and of a real Levi-flat hypersurface, respectively. We say that $\mathcal{F}$ and $M$ are tangent if the leaves of the Levi foliation $\mathcal{L}$ on $M$ are also leaves of $\mathcal{F}$.

The algebraic dimension of Sing $(M)$ is the complex dimension of the singular set of $M_{\mathbb{C}}$.

In the proof of Theorem 1 we will use the following result of [8], which essentially assures that if the singularities of $M$ are sufficiently small (in the algebraic sense) then $M$ is given by the zeroes of the real part of a holomorphic function.

Theorem 2.5 (Cerveau-Lins Neto [8]). Let $M=F^{-1}(0)$ be a germ of an irreducible real analytic Levi-flat hypersurface at $0 \in \mathbb{C}^{n}, n \geq 2$, with Levi 1-form $\eta=i(\partial F-\bar{\partial} F)$. Assume that the algebraic dimension of $\operatorname{Sing}(M)$ is less than or equal to $2 n-4$. Then there exists an unique germ at $0 \in \mathbb{C}^{n}$ of holomorphic codimension-one foliation $\mathcal{F}_{M}$ tangent to $M$, if one of the following conditions is fulfilled:

1. $n \geq 3$ and $\operatorname{cod}_{M_{\mathbb{C}}^{*}}\left(\operatorname{Sing}\left(\left.\eta_{\mathbb{C}}\right|_{M_{\mathbb{C}}^{*}}\right)\right) \geq 3$;

2. $n \geq 2, \operatorname{cod}_{M_{\mathbb{C}}^{*}}\left(\operatorname{Sing}\left(\left.\eta_{\mathbb{C}}\right|_{M_{\mathbb{C}}^{*}}\right) \geq 2\right.$ and $\mathcal{L}_{\mathbb{C}}$ has a non-constant holomorphic first integral. 
Moreover, in both cases the foliation $\mathcal{F}_{M}$ has a non-constant holomorphic first integral $f$ such that $M=(\mathcal{R} e(f)=0)$.

We will assume that the Taylor series of $F$ converges in the polydisk $D_{r}^{n}$. Set $W:=M_{\mathbb{C}}^{*} \backslash \operatorname{Sing}\left(\left.\eta_{\mathbb{C}}\right|_{M_{\mathbb{C}}^{*}}\right)$ and denote by $L_{p}$ the leaf of $\mathcal{L}_{\mathbb{C}}$ through $p$, where $p \in W$.

Lemma 2.6 (Cerveau-Lins Neto [8]). For any $p=\left(z_{0}, w_{0}\right) \in W$ the leaf $L_{p}$ is closed in $M_{\mathbb{C}}^{*}$.

\section{Weighted projective varieties and weighted blow-ups}

In this section we recall the notions of weighted projective space and weighted blow-ups, which will also be used in the proof of Theorem 1. See [9] and [15, page 634] for the basic language and background.

Let $\sigma:=\left(a_{0}, \ldots, a_{n}\right)$ be positive integers. The group $\mathbb{C}^{*}$ acts on $\mathbb{C}^{n+1} \backslash\{0\}$ by

$$
\lambda \cdot\left(x_{0}, \ldots, x_{n}\right)=\left(\lambda^{a_{0}} x_{0}, \ldots, \lambda^{a_{n}} x_{n}\right) .
$$

The quotient space by this action is the weighted projective space of type $\sigma$, $\mathbb{P}\left(a_{0}, \ldots, a_{n}\right):=\mathbb{P}_{\sigma}$. In case $a_{i}>1$ for some $i, \mathbb{P}_{\sigma}$ is a compact algebraic variety with cyclic quotient singularities.

Let $\left[x_{0}: \ldots: x_{n}\right]$ be the homogeneous coordinates on $\mathbb{P}\left(a_{0}, \ldots, a_{n}\right)$. The affine piece $x_{i} \neq 0$ is isomorphic to $\mathbb{C}^{n} / \mathbb{Z}_{a_{i}}$, where $\mathbb{Z}_{a_{i}}$ denotes the quotient group modulo $a_{i}$. Let $\epsilon$ be an $a_{i}^{t h}$-primitive root of unity. The group acts by

$$
z_{j} \longmapsto \epsilon^{a_{j}} z_{j}
$$

for all $j \neq i$, on the coordinates $\left(z_{0}, \ldots, \hat{z_{i}}, \ldots, z_{n}\right)$ of $\mathbb{C}^{n}$; here $z_{j}$ is thought of as $x_{j} / x_{i}^{1 / a_{i}}$. Compare this to the case of $\mathbb{P}^{n}$ where the affine coordinates on $x_{i} \neq 0$ are $z_{j}=x_{j} / x_{i}$.

Definition 3.1. $\mathbb{P}\left(a_{0}, \ldots, a_{n}\right)$ is well-formed if for each $i$

$$
\text { g.c.d. }\left(a_{0}, \ldots, \hat{a}_{i}, \ldots, a_{n}\right)=1 \text {. }
$$

We have a natural orbifold map $\phi_{\sigma}: \mathbb{P}^{n} \rightarrow \mathbb{P}_{\sigma}$ defined by

$$
\left[x_{0}: \ldots: x_{n}\right] \mapsto\left[x_{0}^{a_{0}}: \ldots: x_{n}^{a_{n}}\right]_{\sigma} .
$$

Definition 3.2. Let $X$ be a closed subvariety of a weighted projective space $\mathbb{P}_{\sigma}$, and let $\rho: \mathbb{C}^{n+1} \backslash\{0\} \rightarrow \mathbb{P}_{\sigma}$ be the canonical projection. The punctured affine cone $C_{X}^{*}$ over $X$ is given by $C_{X}^{*}=\rho^{-1}(X)$, and the affine cone $C_{X}$ over $X$ is the completion of $C_{X}^{*}$ in $\mathbb{C}^{n+1}$.

Observe that $\mathbb{C}^{*}$ acts on $C_{X}^{*}$ to give $X=C_{X}^{*} / \mathbb{C}^{*}$.

Lemma 3.3. $C_{X}^{*}$ has no isolated singularities. 
Proof. If $P \in C_{X}^{*}$ is singular then every point on the same fibre of the $\mathbb{C}^{*}$-action is singular.

Definition 3.4. We say that $X$ in $\mathbb{P}_{\sigma}$ is quasi-smooth of dimension $m$ if its affine cone $C_{X}$ is smooth of dimension $m+1$ outside its vertex $0 \in \mathbb{C}^{n+1}$.

When $X \subset \mathbb{P}_{\sigma}$ is quasi-smooth the singularities of $X$ are given by the $\mathbb{C}^{*}$ action and hence are cyclic quotient singularities. Notice that this definition is not equivalent to the smoothness of the inverse image $\phi_{\sigma}^{-1}(X)$ under the quotient map given in (3.1).

Another important fact (cf. [9, Theorem 3.1.6]) is that a quasi-smooth subvariety $X$ of $\mathbb{P}_{\sigma}$ is a $V$-variety, that is, a complex space which is locally isomorphic to the quotient of a complex manifold by a finite group of holomorphic automorphisms.

Now, let $X=\mathbb{C}^{n} / \mathbb{Z}_{m}\left(a_{1}, \ldots, a_{n}\right)$ be a cyclic quotient singularity. That is, $X$ is the quotient variety $\mathbb{C}^{n} / \tau$, where $\tau$ is given by

$$
x_{i} \longmapsto \epsilon^{a_{i}} x_{i}
$$

for all $i$, where $\epsilon$ is an $m^{\text {th }}$-primitive root of unity.

\subsection{Weighted blow-ups}

In this part we will construct the blow-up of $X$. First, we describe $X$ using the theory of toric varieties $(c f$. [14]). Let

$$
e_{1}=(1,0, \ldots, 0), \ldots, e_{n}=(0, \ldots, 0,1) \text { and } e=\frac{1}{m}\left(a_{1}, \ldots, a_{n}\right) .
$$

Then $X=\mathbb{C}^{n} / \mathbb{Z}_{m}\left(a_{1}, \ldots, a_{n}\right)$ is the toric variety corresponding to the lattice $N=$ $\mathbb{Z} e_{1}+\ldots+\mathbb{Z} e_{n}+\mathbb{Z} e$ and the cone $C=\mathbb{R}_{\geq 0} e_{1}+\ldots+\mathbb{R}_{\geq 0} e_{n}$. Denote by $\triangle$ the fan associated to $X$ consisting of all the faces of $C$.

Take $v=\frac{1}{m}\left(a_{1}, \ldots, a_{n}\right) \in N$ with $a_{1}, \ldots, a_{n}>0$ and assume that $e_{1}, \ldots, e_{n}$ and $v$ generate the lattice $N$. Such $v \in N$ will be called a weight. We can construct the weighted blow-up

$$
E: \tilde{X} \rightarrow X=\mathbb{C}^{n} / \mathbb{Z}_{m}\left(a_{1}, \ldots, a_{n}\right)
$$

with weight $v$ as follows: we divide the cone $C$ by adding the 1-dimensional cone $\mathbb{R}_{\geq 0} v$, that is, we divide $C$ into $n$ cones

$$
C_{i}=\mathbb{R}_{\geq 0} e_{1}+\ldots+\mathbb{R}_{\geq 0}^{i-t h} \nu+\ldots+\mathbb{R}_{\geq 0} e_{n} \quad(i=1, \ldots, n) .
$$

Let $\triangle^{\prime}$ be the fan consisting of all the faces of $C_{1}, \ldots, C_{n}$. Then $\tilde{X}$ is the toric variety corresponding to $N$ and $\triangle^{\prime}$ and $E$ is the morphism induced from the natural map of fans $\left(N, \Delta^{\prime}\right) \rightarrow(N, \triangle)$. 
The variety $\tilde{X}$ is covered by $n$ affine open sets $\tilde{U}_{1}, \ldots, \tilde{U}_{n}$ which correspond to the cones $C_{1}, \ldots, C_{n}$ respectively. These affine open sets and $E$ are described as follows:

$$
\begin{gathered}
\tilde{U}_{i}=\mathbb{C}^{n} / \mathbb{Z}_{a_{i}}\left(-a_{1}, \ldots, \stackrel{i^{\text {th }}}{m}, \ldots,-a_{n}\right) \\
\left.E\right|_{\tilde{U}_{i}}: \tilde{U}_{i} \ni\left(y_{1}, \ldots, y_{n}\right) \longmapsto\left(y_{1} y_{i}^{a_{1} / m}, \ldots, y_{i}^{i^{a_{i} / m}}, \ldots, y_{n} y_{i}^{a_{n} / m}\right) \in X .
\end{gathered}
$$

The exceptional divisor $D$ of $E$ is isomorphic to the weighted projective space $\mathbb{P}\left(a_{1}, \ldots, a_{n}\right)$ and $D \cap \tilde{U}_{i}=\left\{y_{i}=0\right\} / \mathbb{Z}_{a_{i}}$.

\section{Theorem 1 in dimension $n \geq 3$}

Theorem 1 will be an immediate consequence of the following proposition. The result is proved in [12], although it is not stated as a separate theorem. We reprove it here for completeness.

Proposition 4.1. Let $Q$ be a quasihomogeneous polynomial with an isolated singularity at $0 \in \mathbb{C}^{n}, n \geq 3$, such that:

1. $F\left(z_{1}, \ldots, z_{n}\right)=\operatorname{Re}\left(Q\left(z_{1}, \ldots, z_{n}\right)\right)+H(z, \bar{z})$, with

$$
H(z, \bar{z})=O\left(|z|^{\operatorname{deg}(Q)+1}\right), H(z, \bar{z})=\bar{H}(\bar{z}, z),
$$

where $\operatorname{deg}(Q)$ is the degree of $Q$ (as a polynomial);

2. $M=F^{-1}(0)$ is Levi-flat.

Then there exists a unique germ at $0 \in \mathbb{C}^{n}$ of holomorphic codimension-one foliation $\mathcal{F}_{M}$ tangent to $M$. Moreover, the foliation $\mathcal{F}_{M}$ has a non-constant holomorphic first integral $f(z)=Q(z)+$ h.o.t. and $M=(\mathcal{R} e(f)=0)$.

Proof. The idea is to use Theorem 2.5 to prove that there exists a germ $f \in \mathcal{O}_{n}$ such that the holomorphic foliation $\mathcal{F}$ defined by $d f=0$ is tangent to $M$ and $M=(\mathcal{R} e(f)=0)$. Note that if $M=(\mathcal{R} e(f)=0)=(F=0)$, with $F \in \mathcal{A}_{n \mathbb{R}}$ irreducible, we must have that $\mathcal{R} e(f)=U F$, where $U \in \mathcal{A}_{n \mathbb{R}}$ and $U(0) \neq 0$. In particular, this implies that $f(z)=Q(z)+$ h.o.t.

Let us prove that we can apply Theorem 2.5. We can write

$$
F(z)=\mathcal{R} e\left(Q\left(z_{1}, \ldots, z_{n}\right)\right)+H(z, \bar{z}),
$$

where $H:\left(\mathbb{C}^{n}, 0\right) \rightarrow(\mathbb{R}, 0)$ is a germ of real-analytic function and $j_{0}^{\operatorname{deg}(Q)}(H)=$ 0 . For simplicity, we assume that $Q$ has real coefficients. Then we get the complexification

$$
F_{\mathbb{C}}(z, w)=\frac{1}{2}(Q(z)+Q(w))+H_{\mathbb{C}}(z, w)
$$

and $M_{\mathbb{C}}=F_{\mathbb{C}}^{-1}(0) \subset\left(\mathbb{C}^{2 n}, 0\right)$. 
Since $Q(z)$ has an isolated singularity at $0 \in \mathbb{C}^{n}$, we get $\operatorname{Sing}\left(M_{\mathbb{C}}\right)=\{0\}$, so the algebraic dimension of Sing $(M)$ is 0 . On other hand, the complexification of $\eta=i(\partial F-\bar{\partial} F)$ is

$$
\eta_{\mathbb{C}}=i\left(\partial_{z} F_{\mathbb{C}}-\partial_{w} F_{\mathbb{C}}\right) .
$$

Recall that $\left.\eta\right|_{M^{*}}$ and $\left.\eta_{\mathbb{C}}\right|_{M_{\mathbb{C}}^{*}}$ define $\mathcal{L}$ and $\mathcal{L}_{\mathbb{C}}$. Now we compute Sing $\left(\left.\eta_{\mathbb{C}}\right|_{M_{\mathbb{C}}^{*}}\right)$ We can write $d F_{\mathbb{C}}=\alpha+\beta$, with

$$
\alpha=\sum_{j=1}^{n} \frac{\partial F_{\mathbb{C}}}{\partial z_{j}} d z_{j}:=\frac{1}{2} \sum_{j=1}^{n}\left(\frac{\partial Q}{\partial z_{j}}(z)+A_{j}\right) d z_{j}
$$

and

$$
\beta=\sum_{j=1}^{n} \frac{\partial F_{\mathbb{C}}}{\partial w_{j}} d w_{j}:=\frac{1}{2} \sum_{j=1}^{n}\left(\frac{\partial Q}{\partial w_{j}}(w)+B_{j}\right) d w_{j},
$$

where $\frac{1}{2} \sum_{j=1}^{n} A_{j} d z_{j}=\sum_{j=1}^{n} \frac{\partial H_{\mathbb{C}}}{\partial z_{j}} d z_{j}$ and $\frac{1}{2} \sum_{j=1}^{n} B_{j} d w_{j}=\sum_{j=1}^{n} \frac{\partial H_{\mathbb{C}}}{\partial w_{j}} d w_{j}$.

Then $\eta_{\mathbb{C}}=i(\alpha-\beta)$, and so

$$
\left.\eta_{\mathbb{C}}\right|_{M_{\mathbb{C}}^{*}}=\left.\left(\eta_{\mathbb{C}}+i d F_{\mathbb{C}}\right)\right|_{M_{\mathbb{C}}^{*}}=\left.2 i \alpha\right|_{M_{\mathbb{C}}^{*}}=-\left.2 i \beta\right|_{M_{\mathbb{C}}^{*}}
$$

In particular, $\left.\alpha\right|_{M_{\mathbb{C}}^{*}}$ and $\left.\beta\right|_{M_{\mathbb{C}}^{*}}$ define $\mathcal{L}_{\mathbb{C}}$. Therefore Sing $\left(\left.\eta_{\mathbb{C}}\right|_{M_{\mathbb{C}}^{*}}\right)$ can be split in two parts. Let $M_{1}=\left\{(z, w) \in M_{\mathbb{C}} \mid \frac{\partial F_{\mathbb{C}}}{\partial w_{j}} \neq 0\right.$ for some $\left.j=1, \ldots, n\right\}$ and $M_{2}=$ $\left\{(z, w) \in M_{\mathbb{C}} \mid \frac{\partial F_{\mathbb{C}}}{\partial z_{j}} \neq 0\right.$ for some $\left.j=1, \ldots, n\right\}$, note that $M_{\mathbb{C}}=M_{1} \cup M_{2}$; if we denote by

$$
X_{1}:=M_{1} \cap\left\{\frac{\partial Q}{\partial z_{1}}(z)+A_{1}=\ldots=\frac{\partial Q}{\partial z_{n}}(z)+A_{n}=0\right\}
$$

and

$$
X_{2}:=M_{2} \cap\left\{\frac{\partial Q}{\partial w_{1}}(w)+B_{1}=\ldots=\frac{\partial Q}{\partial w_{n}}(w)+B_{n}=0\right\},
$$

then Sing $\left(\left.\eta_{\mathbb{C}}\right|_{M_{\mathbb{C}}^{*}}\right)=X_{1} \cup X_{2}$. Since $Q \in \mathbb{C}\left[z_{1}, \ldots, z_{n}\right]$ has an isolated singularity at $0 \in \mathbb{C}^{n}$, we conclude that $\operatorname{cod}_{M_{\mathbb{C}}^{*}} \operatorname{Sing}\left(\left.\eta_{\mathbb{C}}\right|_{M_{\mathbb{C}}^{*}}\right)=n$. If $n \geq 3$, we can directly apply Theorem 2.5 and the proof is complete.

Remark 4.2. The normal forms of $A_{k}, D_{k}, E_{k}$ singularities given by Arnold are complex quasihomogeneous polynomials with an isolated singularity at $0 \in \mathbb{C}^{n}$, and they are stable under deformations. For instance, let us consider $f \in \mathcal{O}_{n}$ of $A_{k}$ type and $g=f+$ h.o.t. Then $g$ is right-equivalent to $f$; i.e. there exists $\varphi \in \operatorname{Diff}\left(\mathbb{C}^{n}, 0\right)$ such that $g \circ \varphi^{-1}=f(c f$. [20, page 32]).

The proposition and remark above imply Theorem 1 for $n \geq 3$ as we will see in the next subsection. 


\subsection{Proof of Theorem 1 for $n \geq 3$}

Let $g$ be a germ at $0 \in \mathbb{C}^{n}$, with $n \geq 3$, of $A_{k}, D_{k}$ or $E_{k}$ type, and $F(z)=$ $\mathcal{R} e(g(z))+H(z, \bar{z})$, where

$$
H(z, \bar{z})=O\left(|z|^{\operatorname{deg}(g)+1}\right), H(z, \bar{z})=\bar{H}(\bar{z}, z) .
$$

Assume that $M=F^{-1}(0)$ is Levi-flat. Since $g$ is a quasihomogenous polynomial with $\mu(g, 0)<\infty$, we can apply Proposition 4.1, so that there exists $f \in \mathcal{O}_{n}$ such that $f(z)=g(z)+$ h.o.t. and $M=(\mathcal{R} e(f)=0)$. According to Remark 4.2, $g$ is stable under deformations then there exists $\varphi \in \operatorname{Diff}\left(\mathbb{C}^{n}, 0\right)$ such that $f \circ \varphi^{-1}=g$. Therefore, $\varphi(M)=(\mathcal{R} e(g)=0)$.

\section{Theorem 1 in dimension two}

Let us consider a special situation that appears in the proof of Theorem 1. Let $Y \subset$ $Z=(x, y, z, w) / \mathbb{Z}_{m}\left(a_{1}, \ldots, a_{4}\right)$ be germ of a $V$-subvariety with unique cyclic quotient singularity at $0 \in \mathbb{C}^{4}$, where $a_{i} \in \mathbb{N}$ are pairwise coprime. Let us consider a codimension-one holomorphic foliation $\mathcal{G}$ on $Y$ with $\operatorname{cod}_{Y^{*}}(\operatorname{Sing} \mathcal{G})=2$. Let $E$ : $\tilde{Z} \rightarrow Z$ be the weighted blow-up with weight $v=\frac{1}{m} \sigma$, where $\sigma=\left(a_{1}, \ldots, a_{4}\right)$. Denote by $\tilde{Y}$ the strict transform of $Y$ by $E$ and by $\tilde{\mathcal{G}}:=E^{*} \mathcal{G}$ the foliation on $\tilde{Y}$.

Suppose $\tilde{Y}$ is smooth and set $\tilde{C}=\tilde{Y} \cap \mathbb{P}_{\sigma}$, where $\mathbb{P}_{\sigma}$ is the exceptional divisor of $E$. Assume that $\tilde{C}$ is invariant by $\tilde{\mathcal{G}}$; i.e., it is a union of leaves and singularities of $\tilde{\mathcal{G}}$. We will assume the following cases:

(i) $\operatorname{Sing}(\tilde{\mathcal{G}}) \cap \operatorname{Sing} \mathbb{P}_{\sigma}=\emptyset$;

(ii) $\operatorname{Sing} \mathbb{P}_{\sigma} \subsetneq \operatorname{Sing}(\tilde{\mathcal{G}})$.

Take $S=\tilde{C} \backslash \operatorname{Sing}(\tilde{\mathcal{G}})$; then $S$ is a smooth leaf of $\tilde{\mathcal{G}}$. Fix $p_{0} \in S \backslash \operatorname{Sing} \mathbb{P}_{\sigma}$ and a transverse section $\sum$ through $p_{0}$ (note that if (ii) holds, we shall only need to take $\left.p_{0} \in S\right)$. Let $G \subset \operatorname{Diff}\left(\sum, p_{0}\right)$ be the holonomy group of the leaf $S$ of $\tilde{\mathcal{G}}$. Since $\operatorname{dim}\left(\sum\right)=1$, we can assume that $G \subset \operatorname{Diff}(\mathbb{C}, 0)$.

Observe that if $p \in \operatorname{Sing} \mathbb{P}_{\sigma}$ and $\zeta$ is a loop around $p$ in the leaf $S_{p}$ of $\tilde{\mathcal{G}}$ through $p$, then the holonomy of $\tilde{\mathcal{G}}$ along $\zeta$ is not the identity, but it is a periodic diffeomorphism. This is consistent with the fact that the local fundamental group of the orbifold $S_{p}$ at $p$ is the cyclic group of finite order. See [7] for more details.

Theorem 5.1. In the above situation, suppose that the following properties are verified:

1. For any $p \in Y^{*} \backslash \operatorname{Sing}(\mathcal{G})$ the leaf $L_{p}$ of $\mathcal{G}$ through $p$ is closed in $Y^{*}$;

2. $g^{\prime}(0)$ is a primitive root of unity, for all $g \in G \backslash\{i d\}$.

Then $\mathcal{G}$ has a non-constant holomorphic first integral. 
Proof. Let $G^{\prime}=\left\{g^{\prime}(0) / g \in G\right\}$ and consider the homomorphism $\phi: G \rightarrow G^{\prime}$ defined by $\phi(g)=g^{\prime}(0)$. We claim that $\phi$ is injective. In fact, assume that $\phi(g)=1$ and suppose by contradiction that $g \neq i d$. In this case $g(z)=z+a z^{r+1}+\ldots$, where $a \neq 0$. According to [17], the pseudo-orbits of this transformation accumulate at $0 \in\left(\sum, 0\right)$, contradicting the fact that the leaves of $\tilde{\mathcal{G}}$ are closed and so the assertion is proved. Now, it suffices to prove that any element $g \in G$ has finite order (cf. [18]). In fact, $\phi(g)=g^{\prime}(0)$ is a root of unity thus $g$ has finite order because $\phi$ is injective. Hence, all transformations of $G$ have finite order and $G$ is linearizable.

This implies that there is a coordinate system $w$ on $\left(\sum, 0\right)$ such that $G=$ $\langle w \rightarrow \lambda w\rangle$, where $\lambda$ is a $d^{\text {th }}$-primitive root of unity (cf. [18]). In particular, $\psi(w)=$ $w^{d}$ is a first integral of $G$, that is $\psi \circ g=\psi$ for any $g \in G$.

Let $\Gamma$ be the union of the separatrices of $\mathcal{G}$ through $0 \in \mathbb{C}^{4}$ and $\tilde{\Gamma}$ be its strict transform under $E$. The first integral $\psi$ can be extended to a first integral $\varphi: \tilde{Y} \backslash \tilde{\Gamma} \rightarrow \mathbb{C}$ by setting

$$
\varphi(q)=\psi\left(\tilde{L}_{q} \cap \sum\right),
$$

where $\tilde{L}_{p}$ denotes the leaf of $\tilde{\mathcal{G}}$ through $q$. Since $\psi$ is bounded (in a compact neighborhood of $0 \in \sum$ ), so is $\varphi$. It follows from Riemann extension theorem that $\varphi$ can be extended holomorphically to $\tilde{\Gamma}$ with $\varphi(\tilde{\Gamma})=0$. This provides the first integral of $\mathcal{G}$.

\subsection{Proof of Theorem 1 in dimension two}

The idea is to use Theorem 2.5. Let us assume for the moment that there exists a foliation $\mathcal{F}_{M}$ with a non-constant holomorphic first integral $f$ and $M=(\mathcal{R} e(f)=$ 0 ). Without loss of generality, we can suppose that $f$ is not a power in $\mathcal{O}_{2}$ so that $\mathcal{R} e(f)$ is irreducible $(c f$. [8, Lemma 2.2]). This implies $\mathcal{R} e(f)=U F$, where $U \in \mathcal{A}_{n \mathbb{R}}$ and $U(0) \neq 0$.

Consider for instance $F(x, y)=\mathcal{R} e\left(x^{2}+y^{k+1}\right)+$ h.o.t. If the Taylor expansion of $f$ at $0 \in \mathbb{C}^{2}$ is

$$
f=\sum_{j \geq 2} f_{j},
$$

where $f_{j}$ is a homogeneous polynomial of degree $j$, then

$$
\mathcal{R} e\left(f_{2}\right)=j_{0}^{2}(\mathcal{R} e(f))=j_{0}^{2}(U F)=U(0) \mathcal{R} e\left(x^{2}\right)
$$

hence $f_{2}=U(0) x^{2}$. Similarly, $f_{k+1}=U(0) y^{k+1}$ so that

$$
f(x, y)=U(0)\left(x^{2}+y^{k+1}\right)+\text { h.o.t. }
$$

Therefore by Remark 4.2 there exists $\varphi \in \operatorname{Diff}\left(\mathbb{C}^{2}, 0\right)$ such that $f \circ \varphi^{-1}=x_{1}^{2}+$ $y_{1}^{k+1}$. Hence, $\varphi(M)=\left(\mathcal{R} e\left(x_{1}^{2}+y_{1}^{k+1}\right)=0\right)$ and this finishes the proof of Theorem 1 . We proceed analogously for the other cases. 
Remark 5.2. Let $M$ be as in Theorem 1, that is, given by

$$
\mathcal{R} e(h(z))+H(z, \bar{z})=0,
$$

where $h(z)$ is a germ at $0 \in \mathbb{C}^{2}$ of $A_{k}, D_{k}$ or $E_{k}$ type. It is easy to check that $M_{\mathbb{C}}$ is complex variety of dimension three with an isolated singularity at $0 \in \mathbb{C}^{4}$ and $\operatorname{cod}{ }_{M_{\mathbb{C}}^{*}} \operatorname{Sing}\left(\left.\eta_{\mathbb{C}}\right|_{M_{\mathbb{C}}^{*}}\right)=2$. Recall that $\mathcal{L}_{\mathbb{C}}$ is defined by $\left.\eta_{\mathbb{C}}\right|_{M_{\mathbb{C}}^{*}}=0$.

The rest of the paper is devoted to proving that we are indeed in the conditions of Theorem 2.5. In all cases the idea is to consider a weighted blow-up $E$ at the singularity and prove that each generator of the holonomy group $G$ of $\tilde{\mathcal{L}}_{\mathbb{C}}:=E^{*} \mathcal{L}_{\mathbb{C}}$ with respect to a leaf has finite order. Now as all leaves are closed (see Lemma 2.6), Theorem 5.1 implies that $\mathcal{L}_{\mathbb{C}}$ has a holomorphic first integral. For convenience, the proof will be divided into the following cases: case $A_{k}$ with $k \geq 2$; case $D_{k}$ with $k \geq 5$; case $E_{6}$; case $E_{7}$ and case $E_{8}$.

\subsection{Case $A_{k}$ with $k \geq 2$}

Let $(x, y) \in \mathbb{C}^{2}$. Write

$$
F(x, y)=\mathcal{R} e\left(x^{2}+y^{k+1}\right)+H(x, y, \bar{x}, \bar{y}),
$$

therefore, the complexification $F_{\mathbb{C}}$ of $F$ can be written as

$$
F_{\mathbb{C}}(x, y, z, w)=\frac{1}{2}\left(x^{2}+y^{k+1}\right)+\frac{1}{2}\left(z^{2}+w^{k+1}\right)+H_{\mathbb{C}}(x, y, z, w)
$$

so that $M_{\mathbb{C}}=F_{\mathbb{C}}^{-1}(0) \subset\left(\mathbb{C}^{4}, 0\right)$ has an isolated singularity at $0 \in \mathbb{C}^{4}$; i.e. the algebraic dimension of $\operatorname{Sing}(M)$ is 0 .

We can define the following algebraic hypersurface on $\mathbb{P}(k+1,2, k+1,2)$

$$
V_{M_{\mathbb{C}}}=\left\{Z_{0}^{2}+Z_{1}^{k+1}+Z_{2}^{2}+Z_{3}^{k+1}=0\right\},
$$

where $\left[Z_{0}: Z_{1}: Z_{2}: Z_{3}\right] \in \mathbb{P}(k+1,2, k+1,2)$. It is not difficult to see that $\operatorname{Sing}\left(M_{\mathbb{C}}\right) \subseteq \operatorname{Sing} V_{M_{\mathbb{C}}}$. Observe that $V_{M_{\mathbb{C}}}$ can be considered as a $V$-subvariety

$$
V_{M_{\mathbb{C}}} \subset Z=\mathbb{C}^{4} / \mathbb{Z}(k+1,2, k+1,2) .
$$

Now we can construct the weighted blow-up $E: \widetilde{Z} \rightarrow Z$ with weight $\sigma=(k+$ $1,2, k+1,2)$. Let $\tilde{M}_{\mathbb{C}}$ be the strict transform of $M_{\mathbb{C}}$ by $E$. We take the exceptional divisor $D \cong \mathbb{P}_{\sigma}$ of $E$ with coordinates $\left(Z_{0}, Z_{1}, Z_{2}, Z_{3}\right) \in \mathbb{C}^{4} \backslash\{0\}$. The intersection of $\tilde{M}_{\mathbb{C}}$ with the divisor $\mathbb{P}_{\sigma}$ is the singular algebraic surface

$$
\tilde{C}:=\tilde{M}_{\mathbb{C}} \cap \mathbb{P}_{\sigma}=\left\{Z_{0}^{2}+Z_{1}^{k+1}+Z_{2}^{2}+Z_{3}^{k+1}=0\right\} .
$$

On the other hand, as we have seen in Remark 2.3 , the foliation $\mathcal{L}_{\mathbb{C}}$ is defined by $\left.\alpha\right|_{M_{\mathbb{C}}^{*}}=0$, where

$$
\alpha=x d x+\frac{(k+1)}{2} y^{k} d y+\theta
$$

and $\theta$ is a 1 -form with $j_{0}^{k}(\theta)=0$. 


\subsubsection{Case $k$ even}

For each $i=1, \ldots, 4$ we have the affine open sets of $E$

$$
\tilde{U}_{i}=\mathbb{C}^{4} / \mathbb{Z}_{a_{i}}\left(-a_{1}, \ldots, 1, \ldots,-a_{4}\right)
$$

where $\sigma=\left(a_{1}, a_{2}, a_{3}, a_{4}\right)$. In $\tilde{U}_{3}$, the blow-up $E$ has the expression

$$
E\left(x_{1}, y_{1}, z_{1}, w_{1}\right)=(x, y, z, w),
$$

where $x=x_{1} z_{1}^{k+1}, y=y_{1} z_{1}^{2}, z=z_{1}^{k+1}, w=w_{1} z_{1}^{2}$ and

$$
D \cap \tilde{U}_{3}=\left\{z_{1}=0\right\} / \mathbb{Z}_{k+1} .
$$

In this chart, the pull-back of $\alpha$ by $E$ is given by

$$
E^{*} \alpha=z_{1}^{2 k+1}\left[(k+1)\left(x_{1}^{2}+y_{1}^{k+1}\right) d z_{1}+x_{1} z_{1} d x_{1}+\frac{(k+1)}{2} z_{1} y_{1}^{k} d y_{1}+z_{1} \theta_{1}\right],
$$

where $\theta_{1}=E^{*} \theta / z_{1}^{2 k+2}$. Therefore, the foliation $\tilde{\mathcal{L}}_{\mathbb{C}}:=E^{*} \mathcal{L}_{\mathbb{C}}$ is defined by $\left.\alpha_{1}\right|_{\tilde{M}_{\mathbb{C}}}=0$, where

$$
\alpha_{1}=(k+1)\left(x_{1}^{2}+y_{1}^{k+1}\right) d z_{1}+x_{1} z_{1} d x_{1}+\frac{(k+1)}{2} z_{1} y_{1}^{k} d y_{1}+z_{1} \theta_{1} .
$$

On the other hand, from (5.2) we have

$$
\tilde{C} \cap \tilde{U}_{3}=\left\{z_{1}=1+x_{1}^{2}+y_{1}^{k+1}+w_{1}^{k+1}=0\right\} / \mathbb{Z}_{k+1},
$$

which implies that $\tilde{C}$ is invariant by $\tilde{\mathcal{L}}_{\mathbb{C}} ;$ i.e., it is a union of leaves and singularities of $\tilde{\mathcal{L}}_{\mathbb{C}}$.

From (5.4) we conclude that the singular set of $\tilde{\mathcal{L}}_{\mathbb{C}}$ is given by

$$
\operatorname{Sing} \tilde{\mathcal{L}}_{\mathbb{C}} \cap \tilde{U}_{3}=\left\{z_{1}=x_{1}^{2}+y_{1}^{k+1}=1+w_{1}^{k+1}=0\right\} / \mathbb{Z}_{k+1} .
$$

In $\tilde{U}_{4}$ we introduce coordinates $\left(x_{2}, y_{2}, z_{2}, w_{2}\right)$ so that $E$ has the following expression

$$
E\left(x_{2}, y_{2}, z_{2}, w_{2}\right)=(x, y, z, w) \text {, }
$$

where $x=x_{2} w_{2}^{k+1}, y=y_{2} w_{2}^{2}, z=z_{2} w_{2}^{k+1}, w=w_{2}^{2}$. In this chart, we have $\tilde{\mathcal{L}}_{\mathbb{C}}$ is defined by $\left.\alpha_{2}\right|_{\tilde{M}_{\mathbb{C}}}=0$, where

$$
\alpha_{2}=(k+1)\left(x_{2}^{2}+y_{2}^{k+1}\right) d w_{2}+x_{2} w_{2} d x_{2}+\frac{(k+1)}{2} w_{2} y_{2}^{k} d y_{2}+w_{2} \beta_{1},
$$


and $\beta_{1}=E^{*} \theta / w_{2}^{2 k+2}$. Moreover,

$$
\operatorname{Sing} \tilde{\mathcal{L}}_{\mathbb{C}} \cap \tilde{U}_{4}=\left\{w_{2}=x_{2}^{2}+y_{2}^{k+1}=z_{2}^{2}+1=0\right\} / \mathbb{Z}_{2} .
$$

Now we claim that $\operatorname{Sing} D \cap \operatorname{Sing} \tilde{\mathcal{L}}_{\mathbb{C}}=\emptyset$, where $D$ is the exceptional divisor of $E$. In fact, on $D \cap \tilde{U}_{3}$ the group acts via

$$
x_{1} \longmapsto x_{1}, \quad y_{1} \longmapsto e^{4 \pi i / k+1} y_{1}, \quad w_{1} \longmapsto e^{4 \pi i / k+1} w_{1}
$$

and on $D \cap \tilde{U}_{4}$ the group acts via

$$
x_{2} \longmapsto e^{(k+1) \pi i} x_{2}, \quad y_{2} \longmapsto y_{2}, \quad z_{2} \longmapsto e^{(k+1) \pi i} z_{2} .
$$

Then

$$
\operatorname{Sing} D \cap \tilde{U}_{3}=\left\{y_{1}=w_{1}=z_{1}=0\right\} / \mathbb{Z}_{k+1}
$$

and

$$
\text { Sing } D \cap \tilde{U}_{4}=\left\{x_{2}=w_{2}=z_{2}=0\right\} / \mathbb{Z}_{2},
$$

hence Sing $D \cap \operatorname{Sing} \tilde{\mathcal{L}}_{\mathbb{C}}=\emptyset$, and so the assertion is proved.

\subsubsection{Case $k$ odd}

Let $\sigma=((k+1) / 2,1,(k+1) / 2,1)$; since $\mathbb{P}_{\sigma}$ is well-formed, let us consider the blow-up $E$ with weight $\sigma$. For each $i=1, \ldots, 4$, we have the affine open sets of $E$,

$$
\tilde{U}_{i}=\mathbb{C}^{4} / \mathbb{Z}_{a_{i}}\left(-a_{1}, \ldots, i^{i^{\text {th }}}, \ldots,-a_{4}\right),
$$

where $\sigma=\left(a_{1}, a_{2}, a_{3}, a_{4}\right)$. In $\tilde{U}_{3}$, the blow-up $E$ has the following expression

$$
E\left(x_{1}, y_{1}, z_{1}, w_{1}\right)=(x, y, z, w),
$$

where $x=x_{1} z_{1}^{(k+1) / 2}, y=y_{1} z_{1}, z=z_{1}^{(k+1) / 2}, w=w_{1} z_{1}$ and

$$
D \cap \tilde{U}_{3}=\left\{z_{1}=0\right\} / \mathbb{Z}_{(k+1) / 2} .
$$

In this chart, the pull-back of $\alpha$ by $E$ is given by

$$
E^{*} \alpha=z_{1}^{k}\left[\frac{(k+1)}{2}\left(x_{1}^{2}+y_{1}^{k+1}\right) d z_{1}+x_{1} z_{1} d x_{1}+\frac{(k+1)}{2} z_{1} y_{1}^{k} d y_{1}+z_{1} \theta_{1}\right],
$$

where $\theta_{1}=E^{*} \theta / z_{1}^{k+1}$. Therefore, the foliation $\tilde{\mathcal{L}}_{\mathbb{C}}:=E^{*} \mathcal{L}_{\mathbb{C}}$ is defined by $\left.\alpha_{1}\right|_{\tilde{M}_{\mathbb{C}}}=0$, where

$$
\alpha_{1}=\frac{(k+1)}{2}\left(x_{1}^{2}+y_{1}^{k+1}\right) d z_{1}+x_{1} z_{1} d x_{1}+\frac{(k+1)}{2} z_{1} y_{1}^{k} d y_{1}+z_{1} \theta_{1} .
$$


We see from (5.2) and (5.8) that $\tilde{C}$ is invariant by $\tilde{\mathcal{L}}_{\mathbb{C}}$. Moreover, the singular set of $\tilde{\mathcal{L}}_{\mathbb{C}}$ is given by

$$
\operatorname{Sing} \tilde{\mathcal{L}}_{\mathbb{C}} \cap \tilde{U}_{3}=\left\{z_{1}=x_{1}^{2}+y_{1}^{k+1}=w_{1}^{k+1}+1=0\right\} / \mathbb{Z}_{(k+1) / 2} .
$$

In $\tilde{U}_{4}$ we introduce coordinates $\left(x_{2}, y_{2}, z_{2}, w_{2}\right)$ so that $E$ has the expression

$$
E\left(x_{2}, y_{2}, z_{2}, w_{2}\right)=(x, y, z, w),
$$

where $x=x_{2} w_{2}^{(k+1) / 2}, y=y_{2} w_{2}, z=z_{2} w_{2}^{(k+1) / 2}, w=w_{2}$. In this chart, $\tilde{\mathcal{L}}_{\mathbb{C}}$ is defined by $\left.\alpha_{2}\right|_{\tilde{M}_{\mathbb{C}}}=0$, where

$$
\alpha_{2}=\frac{(k+1)}{2}\left(x_{2}^{2}+y_{2}^{k+1}\right) d w_{2}+x_{2} w_{2} d x_{2}+\frac{(k+1)}{2} w_{2} y_{2}^{k} d y_{2}+w_{2} \beta_{1},
$$

and $\beta_{1}=E^{*} \theta / w_{2}^{k+1}$. Moreover,

$$
\operatorname{Sing} \tilde{\mathcal{L}}_{\mathbb{C}} \cap \tilde{U}_{4}=\left\{w_{2}=x_{2}^{2}+y_{2}^{k+1}=z_{2}^{2}+1=0\right\} .
$$

As in case of even $k$, it is not difficult to see that $\operatorname{Sing} D \cap \operatorname{Sing} \tilde{\mathcal{L}}_{\mathbb{C}}=\emptyset$.

\subsubsection{End of the proof of case $A_{k}$}

Take $S=\tilde{C} \backslash \operatorname{Sing} \tilde{\mathcal{L}}_{\mathbb{C}}$ so that $S$ is a smooth leaf of $\tilde{\mathcal{L}}_{\mathbb{C}}$. Fix $q_{0} \in S \backslash \operatorname{Sing} D$ and a transversal $\sum$ to $S$.

In the case of even $k$, we can work in the chart $\tilde{U}_{4}$, because of the symmetry of the variables in the definition of the variety $M_{\mathbb{C}}$. Take $q_{0}=(1,0,0,0)$ and the section $\sum=\{(1,0,0, t) \mid t \in \mathbb{C}\}$, parameterized by $t$. Call $G$ the holonomy group of the leaf $S$ of $\hat{\mathcal{L}}_{\mathbb{C}}$ in the section $\sum$. From (5.7), we have that

$$
\operatorname{Sing} \tilde{\mathcal{L}}_{\mathbb{C}} \cap \tilde{U}_{4}=\left\{w_{2}=x_{2}^{2}+y_{2}^{k+1}=z_{2}^{2}+1=0\right\} / \mathbb{Z}_{2} .
$$

For each $j=1,2$, let $\rho_{j}$ be a $2^{t d}$-primitive root of -1 . According to [19], the fundamental group $\pi_{1}\left(S, q_{0}\right)$ can be written in terms of generators and relations as

$$
\pi_{1}\left(S, q_{0}\right)=\left\langle\gamma_{j}, \delta_{j}: \gamma_{j}^{k+1}=\delta_{j}^{2}\right\rangle_{1 \leq j \leq 2}
$$

where for each $j, \gamma_{j}, \delta_{j}$ are two loops that turn around

$$
\left\{w_{2}=x_{2}^{2}+y_{2}^{k+1}=z_{2}-\rho_{j}=0\right\} .
$$

Therefore $G=\left\langle f_{j}, g_{j}\right\rangle_{1 \leq j \leq 2}$, where $f_{j}$ and $g_{j}$ correspond to $\left[\gamma_{j}\right]$ and $\left[\delta_{j}\right]$, respectively. We get from (5.6) that $f_{j}^{\prime}(0)=e^{-2 \pi i / k+1}, g_{j}^{\prime}(0)=e^{-\pi i}$ for all $1 \leq j \leq 2$. 
In the case of odd $k$, we work in the chart $\tilde{U}_{4}$. Take $q_{0}=(1,0,0,0)$ and the section $\sum=\{(1,0,0, t) \mid t \in \mathbb{C}\}$, parameterized by $t$. From (5.11) we have that

$$
\operatorname{Sing} \tilde{\mathcal{L}}_{\mathbb{C}} \cap \tilde{U}_{4}=\left\{w_{2}=x_{2}^{2}+y_{2}^{k+1}=z_{2}^{2}+1=0\right\} .
$$

The group $\pi_{1}\left(S, q_{0}\right)$ can be written in terms of generators and relations as

$$
\pi_{1}\left(S, q_{0}\right)=\left\langle\gamma_{j}, \delta_{j}: \gamma_{j}^{(k+1) / 2} \delta_{j}=\delta_{j} \gamma_{j}^{(k+1) / 2}\right\rangle_{1 \leq j \leq 2}
$$

where for each $j, \gamma_{j}, \delta_{j}$ are two loops that turn around

$$
\left\{w_{2}=x_{2}^{2}+y_{2}^{k+1}=z_{2}-\rho_{j}=0\right\} .
$$

Therefore $G=\left\langle f_{j}, g_{j}\right\rangle_{1 \leq j \leq 2}$, where $f_{j}$ and $g_{j}$ correspond to $\left[\gamma_{i}\right]$ and $\left[\delta_{i}\right]$ respectively. We get from $(5.10)$ that $f_{j}^{\prime}(0)=e^{-4 \pi i / k+1}, g_{j}^{\prime}(0)=1$ for all $1 \leq j \leq 2$.

\subsection{Case $D_{k}$ with $k \geq 5$}

Write

$$
F(x, y)=\mathcal{R} e\left(x^{2} y+y^{k-1}\right)+H(x, y, \bar{x}, \bar{y}) .
$$

The complexification $F_{\mathbb{C}}$ of $F$ can be written as

$$
F_{\mathbb{C}}(x, y, z, w)=\frac{1}{2}\left(x^{2} y+y^{k-1}\right)+\frac{1}{2}\left(z^{2} w+w^{k-1}\right)+H_{\mathbb{C}}(x, y, z, w),
$$

so that $M_{\mathbb{C}}=F_{\mathbb{C}}^{-1}(0) \subset\left(\mathbb{C}^{4}, 0\right)$ has an isolated singularity at $0 \in \mathbb{C}^{4}$; i.e., the algebraic dimension of Sing $\left(M_{\mathbb{C}}\right)$ is 0 .

We can define the following algebraic hypersurface on $\mathbb{P}(k-2,2, k-2,2)$

$$
V_{M_{\mathbb{C}}}=\left\{Z_{0}^{2} Z_{1}+Z_{1}^{k-1}+Z_{2}^{2} Z_{3}+Z_{3}^{k-1}=0\right\},
$$

where $\left[Z_{0}: Z_{1}: Z_{2}: Z_{3}\right] \in \mathbb{P}(k-2,2, k-2,2)$. It is not difficult to see that Sing $\left(M_{\mathbb{C}}\right) \subseteq \operatorname{Sing} V_{M_{\mathbb{C}}}$. Note that $V_{M_{\mathbb{C}}}$ can be considered as a $V$-subvariety

$$
V_{M_{\mathbb{C}}} \subset Z=\mathbb{C}^{4} / \mathbb{Z}(k-2,2, k-2,2) .
$$

We consider the weighted blow-up $E: \widetilde{Z} \rightarrow Z$ with weight $\sigma=(k-2,2, k-2,2)$. Let $\tilde{M}_{\mathbb{C}}$ be the strict transform of $M_{\mathbb{C}}$ by $E$. We take the exceptional divisor $D \cong \mathbb{P}_{\sigma}$ of $E$ with coordinates $\left(Z_{0}, Z_{1}, Z_{2}, Z_{3}\right) \in \mathbb{C}^{4} \backslash\{0\}$. The intersection of $\tilde{M}_{\mathbb{C}}$ with the divisor $\mathbb{P}_{\sigma}$ is the singular algebraic surface

$$
\tilde{C}:=\tilde{M}_{\mathbb{C}} \cap \mathbb{P}_{\sigma}=\left\{Z_{0}^{2} Z_{1}+Z_{1}^{k-1}+Z_{2}^{2} Z_{3}+Z_{3}^{k-1}=0\right\} .
$$

On the other hand, as we have seen in Remark 2.3 , the foliation $\mathcal{L}_{\mathbb{C}}$ is defined by $\left.\alpha\right|_{M_{\mathbb{C}}^{*}}=0$, where

$$
\alpha=x y d x+\frac{1}{2}\left(x^{2}+(k-1) y^{k-2}\right) d y+\theta
$$

and $\theta$ is a 1 -form with $j_{0}^{k-2}(\theta)=0$. 


\subsubsection{Case $k$ even}

Let $\sigma=((k-2) / 2,1,(k-2) / 2,1)$; since $\mathbb{P}_{\sigma}$ is well-formed, let us consider $E$ with weight $\sigma$. For each $i=1, \ldots, 4$, we have the affine open sets of $E$,

$$
\tilde{U}_{i}=\mathbb{C}^{4} / \mathbb{Z}_{a_{i}}\left(-a_{1}, \ldots, \stackrel{i^{\text {th }}}{1, \ldots,-a_{4}}\right),
$$

where $\sigma=\left(a_{1}, a_{2}, a_{3}, a_{4}\right)$. In $\tilde{U}_{3}$, the blow-up $E$ has the expression

$$
E\left(x_{1}, y_{1}, z_{1}, w_{1}\right)=(x, y, z, w),
$$

where $x=x_{1} z_{1}^{(k-2) / 2}, y=y_{1} z_{1}, z=z_{1}^{(k-2) / 2}, w=w_{1} z_{1}$ and

$$
D \cap \tilde{U}_{3}=\left\{z_{1}=0\right\} / \mathbb{Z}_{(k-2) / 2} .
$$

In this chart, the pull-back of $\alpha$ by $E$ is given by

$$
\begin{array}{r}
E^{*} \alpha=z_{1}^{k-2}\left[\frac{(k-1)}{2}\left(x_{1}^{2} y_{1}+y_{1}^{k-1}\right) d z_{1}+x_{1} y_{1} z_{1} d x_{1}\right. \\
\left.+\frac{1}{2}\left(x_{1}^{2}+(k-1) y_{1}^{k-2}\right) z_{1} d y_{1}+z_{1} \theta_{1}\right],
\end{array}
$$

where $\theta_{1}=E^{*} \theta / z_{1}^{k-1}$. Therefore, the foliation $\tilde{\mathcal{L}}_{\mathbb{C}}:=E^{*} \mathcal{L}_{\mathbb{C}}$ is defined by $\left.\alpha_{1}\right|_{\tilde{M}_{\mathbb{C}}}=0$, where

$$
\begin{aligned}
\alpha_{1}= & \frac{(k-1)}{2}\left(x_{1}^{2} y_{1}+y_{1}^{k-1}\right) d z_{1}+x_{1} y_{1} z_{1} d x_{1} \\
& +\frac{1}{2}\left(x_{1}^{2}+(k-1) y_{1}^{k-2}\right) z_{1} d y_{1}+z_{1} \theta_{1} .
\end{aligned}
$$

From (5.13) we have

$$
\tilde{C} \cap \tilde{U}_{3}=\left\{z_{1}=x_{1}^{2} y_{1}+y_{1}^{k-1}+w_{1}+w_{1}^{k-1}=0\right\} / \mathbb{Z}_{(k-2) / 2}
$$

which implies that $\tilde{C}$ is invariant by $\tilde{\mathcal{L}}_{\mathbb{C}}$. Now from (5.15) we deduce that the singular set of $\tilde{\mathcal{L}}_{\mathbb{C}}$ is given by

$$
\text { Sing } \tilde{\mathcal{L}}_{\mathbb{C}} \cap \tilde{U}_{3}=\left\{z_{1}=x_{1}^{2} y_{1}+y_{1}^{k-1}=w_{1}+w_{1}^{k-1}=0\right\} / \mathbb{Z}_{(k-2) / 2} .
$$

In $\tilde{U}_{4}$ we introduce coordinates $\left(x_{2}, y_{2}, z_{2}, w_{2}\right)$ so that $E$ has the expression

$$
E\left(x_{2}, y_{2}, z_{2}, w_{2}\right)=(x, y, z, w)
$$


where $x=x_{2} w_{2}^{(k-2) / 2}, y=y_{2} w_{2}, z=z_{2} w_{2}^{(k-2) / 2}, w=w_{2}$. In this chart, we have that $\tilde{\mathcal{L}}_{\mathbb{C}}$ is defined by $\left.\alpha_{2}\right|_{\tilde{M}_{\mathbb{C}}}=0$, where

$$
\begin{aligned}
\alpha_{2}= & \frac{(k-1)}{2}\left(x_{2}^{2} y_{2}+y_{2}^{k-1}\right) d w_{2}+x_{2} y_{2} w_{2} d x_{2} \\
& +\frac{1}{2}\left(x_{2}^{2}+(k-1) y_{2}^{k-2}\right) w_{2} d y_{2}+w_{2} \beta_{1},
\end{aligned}
$$

and $\beta_{1}=E^{*} \theta / \bar{w}_{1}^{k-1}$. Moreover,

$$
\operatorname{Sing} \tilde{\mathcal{L}}_{\mathbb{C}} \cap \tilde{U}_{4}=\left\{w_{2}=x_{2}^{2} y_{2}+y_{2}^{k-1}=z_{2}^{2}+1=0\right\} .
$$

We claim that $\operatorname{Sing} D \subsetneq \operatorname{Sing} \tilde{\mathcal{L}}_{\mathbb{C}}$, where $D$ is the exceptional divisor of $E$. In fact, on $D \cap \tilde{U}_{3}$ the group acts via

$$
x_{1} \longmapsto x_{1}, \quad y_{1} \longmapsto e^{4 \pi i / k-2} y_{1}, \quad w_{1} \longmapsto e^{4 \pi i / k-2} w_{1} .
$$

Since $k$ is even, $\operatorname{Sing} D \cap \tilde{U}_{4}=\emptyset$, so

$$
\operatorname{Sing} D \cap \tilde{U}_{3}=\left\{y_{1}=z_{1}=w_{1}=0\right\} / \mathbb{Z}_{(k-2) / 2} .
$$

Note that it is an irreducible component of Sing $\tilde{\mathcal{L}}_{\mathbb{C}}$ and so the assertion is proved.

5.3.2. Case $k$ odd

Let us consider $E$ with weight $\sigma=(k-2,2, k-2,2)$. For each $i=1, \ldots, 4$, we have the affine open sets of $E$,

$$
\tilde{U}_{i}=\mathbb{C}^{4} / \mathbb{Z}_{a_{i}}\left(-a_{1}, \ldots, i^{i^{\mathrm{th}}}, \ldots,-a_{4}\right),
$$

where $\sigma=\left(a_{1}, a_{2}, a_{3}, a_{4}\right)$. In $\tilde{U}_{3}$, the blow-up $E$ has the expression:

$$
E\left(x_{1}, y_{1}, z_{1}, w_{1}\right)=(x, y, z, w) \text {, }
$$

where $x=x_{1} z_{1}^{k-2}, y=y_{1} z_{1}^{2}, z=z_{1}^{k-2}, w=w_{1} z_{1}^{2}$ and

$$
D \cap \tilde{U}_{3}=\left\{z_{1}=0\right\} / \mathbb{Z}_{k-2} \text {. }
$$

In this chart, the pull-back of $\alpha$ by $E$ is given by

$$
\begin{aligned}
E^{*} \alpha=z_{1}^{2 k-3}[ & (k-1)\left(x_{1}^{2} y_{1}+y_{1}^{k-1}\right) d z_{1}+x_{1} y_{1} z_{1} d x_{1} \\
& \left.+\frac{1}{2}\left(x_{1}^{2}+(k-1) y_{1}^{k-2}\right) z_{1} d y_{1}+z_{1} \theta_{1}\right]
\end{aligned}
$$


where $\theta_{1}=E^{*} \theta / z_{1}^{2 k-4}$. Therefore, the foliation $\tilde{\mathcal{L}}_{\mathbb{C}}:=E^{*} \mathcal{L}_{\mathbb{C}}$ is defined by $\left.\alpha_{1}\right|_{\tilde{M}_{\mathbb{C}}}=0$, where

$$
\begin{aligned}
\alpha_{1}= & (k-1)\left(x_{1}^{2} y_{1}+y_{1}^{k-1}\right) d z_{1}+x_{1} y_{1} z_{1} d x_{1} \\
& +\frac{1}{2}\left(x_{1}^{2}+(k-1) y_{1}^{k-2}\right) z_{1} d y_{1}+z_{1} \theta_{1} .
\end{aligned}
$$

From (5.13) we have

$$
\tilde{C} \cap \tilde{U}_{3}=\left\{z_{1}=x_{1}^{2} y_{1}+y_{1}^{k-1}+w_{1}+w_{1}^{k-1}=0\right\} / \mathbb{Z}_{k-2}
$$

which implies that $\tilde{C}$ is invariant by $\tilde{\mathcal{L}}_{\mathbb{C}}$. Now from (5.15) we deduce that the singular set of $\tilde{\mathcal{L}}_{\mathbb{C}}$ is given by

$$
\text { Sing } \tilde{\mathcal{L}}_{\mathbb{C}} \cap \tilde{U}_{3}=\left\{z_{1}=x_{1}^{2} y_{1}+y_{1}^{k-1}=w_{1}+w_{1}^{k-1}=0\right\} / \mathbb{Z}_{k-2} .
$$

In $\tilde{U}_{4}$ we introduce coordinates $\left(x_{2}, y_{2}, z_{2}, w_{2}\right)$ so that $E$ has the expression

$$
E\left(x_{2}, y_{2}, z_{2}, w_{2}\right)=(x, y, z, w),
$$

where $x=x_{2} w_{2}^{k-2}, y=y_{2} w_{2}^{2}, z=z_{2} w_{2}^{k-2}, w=w_{2}^{2}$ and

$$
D \cap \tilde{U}_{3}=\left\{w_{2}=0\right\} / \mathbb{Z}_{2} .
$$

In this chart, we have $\tilde{\mathcal{L}}_{\mathbb{C}}$ is defined by $\left.\alpha_{2}\right|_{\tilde{M}_{\mathbb{C}}}=0$, where

$$
\begin{aligned}
\alpha_{2}= & (k-1)\left(x_{2}^{2} y_{2}+y_{2}^{k-1}\right) d w_{2}+x_{2} y_{2} w_{2} d x_{2} \\
& +\frac{1}{2}\left(x_{2}^{2}+(k-1) y_{2}^{k-2}\right) w_{2} d y_{2}+w_{2} \beta_{1},
\end{aligned}
$$

and $\beta_{1}=E^{*} \theta / w_{2}^{2 k-4}$. Moreover,

$$
\text { Sing } \tilde{\mathcal{L}}_{\mathbb{C}} \cap \tilde{U}_{4}=\left\{w_{2}=x_{2}^{2} y_{2}+y_{2}^{k-1}=z_{2}^{2}+1=0\right\} / \mathbb{Z}_{2} .
$$

Now we assert that $\operatorname{Sing} D \subsetneq \operatorname{Sing} \tilde{\mathcal{L}}_{\mathbb{C}}$, where $D$ is the exceptional divisor of $E$. In fact, on $D \cap \tilde{U}_{3}$ the group acts via

$$
x_{1} \longmapsto x_{1}, \quad y_{1} \longmapsto e^{4 \pi i / k-2} y_{1}, \quad w_{1} \longmapsto e^{4 \pi i / k-2} w_{1}
$$

and on $D \cap \tilde{U}_{4}$ the group acts via

$$
x_{2} \longmapsto e^{(k-2) \pi i} x_{2}, \quad y_{2} \longmapsto y_{2}, \quad z_{2} \longmapsto e^{(k-2) \pi i} z_{2} .
$$

Therefore

$$
\text { Sing } D \cap \tilde{U}_{3}=\left\{y_{1}=z_{1}=w_{1}=0\right\} / \mathbb{Z}_{k-2}
$$

is an irreducible component of Sing $\tilde{\mathcal{L}}_{\mathbb{C}}$ and

$$
\operatorname{Sing} D \cap \tilde{U}_{4}=\left\{x_{2}=z_{2}=w_{2}=0\right\} / \mathbb{Z}_{2}
$$

does not intersect the singular set of $\tilde{\mathcal{L}}_{\mathbb{C}}$, so the assertion is proved. 


\subsubsection{End of the proof of case $D_{k}$}

Take $S=\tilde{C} \backslash \operatorname{Sing} \tilde{\mathcal{L}}_{\mathbb{C}}$, so that $S$ is a smooth leaf of $\tilde{\mathcal{L}}_{\mathbb{C}}$. Fix $q_{0} \in S$ and a transversal $\sum$ to $S$. Observe that the above assertion implies that $q_{0} \notin \operatorname{Sing} D$.

In the case of even $k$, we work in the chart $\tilde{U}_{4}$. Take $q_{0}=(1,0,0,0)$ and the section $\sum=\{(1,0,0, t) \mid t \in \mathbb{C}\}$, parameterized by $t$. Call $G$ the holonomy group of the leaf $S$ of $\tilde{\mathcal{L}}_{\mathbb{C}}$ in the section $\sum$. From (5.18), we have that

$$
\text { Sing } \tilde{\mathcal{L}}_{\mathbb{C}} \cap \tilde{U}_{4}=\left\{w_{2}=x_{2}^{2} y_{2}+y_{2}^{k-1}=z_{2}^{2}+1=0\right\} .
$$

For each $j=1,2$, denote by $r_{j}$ a $2^{t d}$-primitive root of -1 . The group $\pi_{1}\left(S, q_{0}\right)$ can be written in terms of generators and relations as

$$
\pi_{1}\left(S, q_{0}\right)=\left\langle\gamma_{j}, \delta_{j}, \zeta_{j}: \gamma_{j}^{(k-2) / 2} \delta_{j}=\delta_{j} \gamma_{j}^{(k-2) / 2}\right\rangle_{1 \leq j \leq 2}
$$

where for each $j, \gamma_{j}, \delta_{j}$ are loops that turn around

$$
\left\{w_{2}=x_{2}^{2}+y_{2}^{k-2}=z_{2}-r_{j}=0\right\},
$$

and $\zeta_{j}$ are loops that turn around $\left\{w_{2}=y_{2}=z_{2}-r_{j}=0\right\}$ Therefore $G=$ $\left\langle f_{j}, g_{j}, h_{j}\right\rangle_{1 \leq j \leq 2}$, where $f_{j}, g_{j}$ and $h_{j}$ correspond to $\left[\gamma_{j}\right],\left[\delta_{j}\right]$ and $\left[\zeta_{j}\right]$ respectively. We get from (5.17) that $f_{j}^{\prime}(0)=e^{-4 \pi i / k-2}, g_{j}^{\prime}(0)=1$ and $h_{j}^{\prime}(0)=1$ for all $1 \leq j \leq 2$.

In the case of odd $k$, we work in the chart $\tilde{U}_{4}$. Take $q_{0}=(1,0,0,0)$ and the section $\sum=\{(1,0,0, t) \mid t \in \mathbb{C}\}$, parameterized by $t$. From (5.22) we have that

$$
\operatorname{Sing} \tilde{\mathcal{L}}_{\mathbb{C}} \cap \tilde{U}_{4}=\left\{w_{2}=x_{2}^{2} y_{2}+y_{2}^{k-1}=z_{2}^{2}+1=0\right\} / \mathbb{Z}_{2} .
$$

The fundamental group $\pi_{1}\left(S, q_{0}\right)$ is generated by

$$
\pi_{1}\left(S, q_{0}\right)=\left\langle\gamma_{j}, \delta_{j}, \zeta_{j}: \gamma_{j}^{k-2}=\delta_{j}^{2}\right\rangle_{1 \leq j \leq 2},
$$

where for each $j, \gamma_{j}, \delta_{j}$ are loops that turn around

$$
\left\{w_{2}=x_{2}^{2}+y_{2}^{k-2}=z_{2}-r_{j}=0\right\},
$$

$\zeta_{j}$ are loops that turn around $\left\{w_{2}=y_{2}=z_{2}-r_{j}=0\right\}$. Therefore $G=$ $\left\langle f_{j}, g_{j}, h_{j}\right\rangle_{1 \leq j \leq 2}$, where $f_{j}, g_{j}$ and $h_{j}$ correspond to $\left[\gamma_{i}\right],\left[\delta_{i}\right]$ and $\left[\zeta_{i}\right]$ respectively. We get from (5.17) that $f_{j}^{\prime}(0)=e^{-2 \pi i / k-2}, g_{j}^{\prime}(0)=e^{-\pi i}$ and $h_{j}^{\prime}(0)=1$ for all $1 \leq j \leq 2$. 


\subsection{Case $E_{6}$}

Write

$$
F(x, y)=\mathcal{R} e\left(x^{4}+y^{3}\right)+H(x, y, \bar{x}, \bar{y}) .
$$

The complexification $F_{\mathbb{C}}$ of $F$ can be written as

$$
F_{\mathbb{C}}(x, y, z, w)=\frac{1}{2}\left(x^{4}+y^{3}\right)+\frac{1}{2}\left(z^{4}+w^{3}\right)+H_{\mathbb{C}}(x, y, z, w),
$$

so that $M_{\mathbb{C}}=F_{\mathbb{C}}^{-1}(0) \subset\left(\mathbb{C}^{4}, 0\right)$. Note that $0 \in \mathbb{C}^{4}$ is an isolated singularity of $M_{\mathbb{C}}$ so the algebraic dimension of $\operatorname{Sing} M$ is 0 .

Let us define the following algebraic hypersurface on $\mathbb{P}(3,4,3,4)$

$$
V_{M_{\mathbb{C}}}:=\left\{Z_{0}^{4}+Z_{1}^{3}+Z_{2}^{4}+Z_{3}^{3}=0\right\},
$$

where $\left[Z_{0}: Z_{1}: Z_{2}: Z_{3}\right] \in \mathbb{P}(3,4,3,4)$. Clearly Sing $M_{\mathbb{C}} \subset$ Sing $V_{M_{\mathbb{C}}}$ and $V_{M_{\mathbb{C}}}$ can be considered as a $V$-subvariety

$$
V_{M_{\mathbb{C}}} \subset Z=\mathbb{C}^{4} / \mathbb{Z}(3,4,3,4)
$$

Let $E: \tilde{Z} \rightarrow Z$ be the weighted blow-up with weight $\sigma=(3,4,3,4)$. Denote by $\tilde{M}_{\mathbb{C}}$ the strict transform of $M_{\mathbb{C}}$ under $E$. Take the exceptional divisor $D \cong \mathbb{P}_{\sigma}$ of $E$ with coordinates $\left(Z_{0}, Z_{1}, Z_{2}, Z_{3}\right) \in \mathbb{C}^{4} \backslash\{0\}$. The intersection of $\tilde{M}_{\mathbb{C}}$ with $\mathbb{P}_{\sigma}$ is the algebraic surface

$$
\tilde{C}=\tilde{M}_{\mathbb{C}} \cap \mathbb{P}_{\sigma}=\left\{Z_{0}^{4}+Z_{1}^{3}+Z_{2}^{4}+Z_{3}^{3}=0\right\} .
$$

On the other hand, according to Remark (2.3), the foliation $\mathcal{L}_{\mathbb{C}}$ is defined by $\left.\alpha\right|_{M_{\mathbb{C}}^{*}}=$ 0 , where

$$
\alpha=2 x^{3} d x+\frac{3}{2} y^{2} d y+\theta,
$$

where $\theta$ is a 1 -form with $j_{0}^{3}(\theta)=0$. For each $i=1, \ldots, 4$, we have the affine open sets of $E$

$$
\tilde{U}_{i}=\mathbb{C}^{4} / \mathbb{Z}_{a_{i}}\left(-a_{1}, \ldots, 1, \ldots,-a_{4}\right)
$$

where $\sigma=\left(a_{1}, a_{2}, a_{3}, a_{4}\right)$. In $\tilde{U}_{3}$, the blow-up $E$ has the expression:

$$
E\left(x_{1}, y_{1}, z_{1}, w_{1}\right)=(x, y, z, w),
$$

where $x=x_{1} z_{1}^{3}, y=y_{1} z_{1}^{4}, z=z_{1}^{3}, w=w_{1} z_{1}^{4}$ and $D \cap \tilde{U}_{3}=\left\{z_{1}=0\right\} / \mathbb{Z}_{3}$.

In this chart, the pull-back of $\alpha$ by $E$ is given by

$$
E^{*} \alpha=z_{1}^{11}\left[6\left(x_{1}^{4}+y_{1}^{3}\right) d z_{1}+2 z_{1} x_{1}^{3} d x_{1}+\frac{3}{2} z_{1} y_{1}^{2} d y_{1}+z_{1} \theta_{1}\right]
$$


where $\theta_{1}=E^{*} \alpha / z_{1}^{12}$. Therefore the foliation $\tilde{\mathcal{L}}_{\mathbb{C}}:=E^{*} \mathcal{L}_{\mathbb{C}}$ is defined by $\left.\alpha_{1}\right|_{\tilde{M}_{\mathbb{C}}}=$ 0 , where

$$
\alpha_{1}=6\left(x_{1}^{4}+y_{1}^{3}\right) d z_{1}+2 z_{1} x_{1}^{3} d x_{1}+\frac{3}{2} z_{1} y_{1}^{2} d y_{1}+z_{1} \theta_{1} .
$$

From (5.24) we have

$$
\tilde{C} \cap \tilde{U}_{3}=\left\{z_{1}=x_{1}^{4}+y_{1}^{3}+w_{1}^{3}+1=0\right\} / \mathbb{Z}_{3}
$$

which implies that $\tilde{C}$ is invariant by $\tilde{\mathcal{L}}_{\mathbb{C}}$. Now it follows from (5.26) that the singular set of $\tilde{\mathcal{L}}_{\mathbb{C}}$ is given by

$$
\text { Sing } \tilde{\mathcal{L}}_{\mathbb{C}} \cap \tilde{U}_{3}=\left\{z_{1}=x_{1}^{4}+y_{1}^{3}=w_{1}^{3}+1=0\right\} / \mathbb{Z}_{3} .
$$

In $\tilde{U}_{4}$ we introduce coordinates $\left(x_{2}, y_{2}, z_{2}, w_{2}\right)$ so that $E$ has the expression

$$
E\left(x_{2}, y_{2}, z_{2}, w_{2}\right)=(x, y, z, w),
$$

where $x=x_{2} w_{2}^{3}, y=y_{2} w_{2}^{4}, z=z_{2} w_{2}^{3}, w=w_{2}^{4}$ and $D \cap \tilde{U}_{4}=\left\{w_{2}=0\right\} / \mathbb{Z}_{4}$. In this chart, the foliation $\tilde{\mathcal{L}}_{\mathbb{C}}$ is defined by $\left.\alpha_{2}\right|_{\tilde{M}_{\mathbb{C}}}=0$, where

$$
\alpha_{2}=6\left(x_{2}^{4}+y_{2}^{3}\right) d w_{2}+2 w_{2} x_{2}^{3} d x_{2}+\frac{3}{2} w_{2} y_{2}^{2} d y_{2}+w_{2} \beta_{1},
$$

and $\beta_{1}=E^{*} \theta / w_{2}^{12}$. Moreover

$$
\operatorname{Sing} \tilde{\mathcal{L}}_{\mathbb{C}} \cap \tilde{U}_{4}=\left\{w_{2}=x_{2}^{4}+y_{2}^{3}=z_{2}^{4}+1=0\right\} / \mathbb{Z}_{4} .
$$

We assert that Sing $D \cap \operatorname{Sing} \tilde{\mathcal{L}}_{\mathbb{C}}=\emptyset$, where $D \cong \mathbb{P}(3,4,3,4)$ is the exceptional divisor of $E$. In fact, on $D \cap \tilde{U}_{3}$ the group acts via

$$
x_{1} \longmapsto x_{1}, \quad y_{1} \longmapsto e^{8 \pi i / 3} y_{1}, \quad w_{1} \longmapsto e^{8 \pi i / 3} w_{1}
$$

and on $D \cap \tilde{U}_{4}$ the group acts via

$$
x_{2} \longmapsto e^{3 \pi i / 2} x_{2}, \quad y_{2} \longmapsto y_{2}, \quad z_{2} \longmapsto e^{3 \pi i / 2} z_{2} .
$$

Therefore

$$
\text { Sing } D \cap \tilde{U}_{3}=\left\{y_{1}=z_{1}=w_{1}=0\right\} / \mathbb{Z}_{3}
$$

and

$$
\text { Sing } D \cap \tilde{U}_{4}=\left\{x_{2}=z_{2}=w_{2}=0\right\} / \mathbb{Z}_{4},
$$

hence Sing $\tilde{\mathcal{L}}_{\mathbb{C}} \cap \operatorname{Sing} D=\emptyset$, so the assertion is proved. 


\subsubsection{End of the proof of case $E_{6}$}

Take $S=\tilde{C} \backslash \operatorname{Sing} \tilde{\mathcal{L}}_{\mathbb{C}}$ so that $S$ is a smooth leaf of $\tilde{\mathcal{L}}_{\mathbb{C}}$. Fix $q_{0} \in S \backslash \operatorname{Sing} D$ and a transversal $\sum$ to $S$.

We work in the chart $\tilde{U}_{3}$. Take $q_{0}=(1,0,0,0)$ and the section $\sum=\{(1,0, t, 0) \mid t \in$ $\mathbb{C}$ \}, parameterized by $t$. Call $G$ the holonomy group of the leaf $S$ of $\hat{\mathcal{L}}_{\mathbb{C}}$ in the section $\sum$. From (5.27), we have

$$
\text { Sing } \tilde{\mathcal{L}}_{\mathbb{C}} \cap \tilde{U}_{3}=\left\{z_{1}=x_{1}^{4}+y_{1}^{3}=w_{1}^{3}+1=0\right\} / \mathbb{Z}_{3} .
$$

For each $j=1,2,3$, denote by $\rho_{j}$ a $3^{\text {td }}$-primitive root of -1 . The group $\pi_{1}\left(S, q_{0}\right)$ can be written in terms of generators and relations as

$$
\pi\left(S, q_{0}\right)=\left\langle\gamma_{j}, \zeta_{j}: \gamma_{j}^{3}=\zeta_{j}^{4}\right\rangle_{1 \leq j \leq 3}
$$

where $\gamma_{j}, \zeta_{j}$ are loops that turn around

$$
\left\{z_{1}=x_{1}^{4}+y_{1}^{3}=w_{1}-\rho_{j}=0\right\}, \text { for all } 1 \leq j \leq 3 .
$$

Therefore $G=\left\langle f_{j}, g_{j}\right\rangle_{1 \leq j \leq 3}$, where $f_{j}$ and $g_{j}$ correspond to $\left[\gamma_{j}\right]$ and $\left[\zeta_{j}\right]$ respectively. We get from (5.26) that $f_{j}^{\prime}(0)=e^{-2 \pi i / 3}, g_{j}^{\prime}(0)=e^{-\pi i / 2}$, for all $1 \leq j \leq 3$.

\subsection{Case $E_{7}$}

Let us consider

$$
F(x, y)=\mathcal{R} e\left(x^{3} y+y^{3}\right)+H(x, y, \bar{x}, \bar{y}),
$$

therefore, the complexification $F_{\mathbb{C}}$ of $F$ can be written as

$$
F_{\mathbb{C}}(x, y, z, w)=\frac{1}{2}\left(x^{3} y+y^{3}\right)+\frac{1}{2}\left(z^{3} w+w^{3}\right)+H_{\mathbb{C}}(x, y, z, w),
$$

so that $M_{\mathbb{C}}=F_{\mathbb{C}}^{-1}(0) \subset\left(\mathbb{C}^{4}, 0\right)$. Note that $0 \in \mathbb{C}^{4}$ is an isolated singularity of $M_{\mathbb{C}}$ so the algebraic dimension of $\operatorname{Sing} M$ is 0 .

Let us define the following algebraic hypersurface on $\mathbb{P}(2,3,2,3)$

$$
V_{M_{\mathbb{C}}}:=\left\{Z_{0}^{3} Z_{1}+Z_{1}^{3}+Z_{2}^{3} Z_{3}+Z_{3}^{3}=0\right\},
$$

where $\left[Z_{0}: Z_{1}: Z_{2}: Z_{3}\right] \in \mathbb{P}(2,3,2,3)$. Clearly Sing $M_{\mathbb{C}} \subset \operatorname{Sing} V_{M_{\mathbb{C}}}$ and $V_{M_{\mathbb{C}}}$ can be considered as a $V$-subvariety

$$
V_{M_{\mathbb{C}}} \subset Z=\mathbb{C}^{4} / \mathbb{Z}(2,3,2,3) .
$$

Let $E: \tilde{Z} \rightarrow Z$ be the weighted blow-up with weight $\sigma=(2,3,2,3)$. Denote by $\tilde{M}_{\mathbb{C}}$ the strict transform of $M_{\mathbb{C}}$ by $E$. Take the exceptional divisor $D \cong \mathbb{P}_{\sigma}$ of $E$ 
with coordinates $\left(Z_{0}, Z_{1}, Z_{2}, Z_{3}\right) \in \mathbb{C}^{4} \backslash\{0\}$. The intersection of $\tilde{M}_{\mathbb{C}}$ with $\mathbb{P}_{\sigma}$ is the algebraic surface

$$
\tilde{C}=\tilde{M}_{\mathbb{C}} \cap \mathbb{P}_{\sigma}=\left\{Z_{0}^{3} Z_{1}+Z_{1}^{3}+Z_{2}^{3} Z_{3}+Z_{3}^{3}=0\right\} .
$$

On the other hand, according to Remark (2.3), the foliation $\mathcal{L}_{\mathbb{C}}$ is defined by $\left.\alpha\right|_{M_{\mathbb{C}}^{*}}=$ 0 , where

$$
\alpha=\frac{3}{2} x^{2} y d x+\frac{1}{2}\left(x^{3}+3 y^{2}\right) d y+\theta
$$

where $\theta$ is a 1 -form with $j_{0}^{3}(\theta)=0$. For each $i=1, \ldots, 4$, we have the affine open sets of $E$

$$
\tilde{U}_{i}=\mathbb{C}^{4} / \mathbb{Z}_{a_{i}}\left(-a_{1}, \ldots, i^{\text {th }}, \ldots,-a_{4}\right),
$$

where $\sigma=\left(a_{1}, a_{2}, a_{3}, a_{4}\right)$. In $\tilde{U}_{3}$, the blow-up $E$ has the expression

$$
E\left(x_{1}, y_{1}, z_{1}, w_{1}\right)=(x, y, z, w),
$$

where $x=x_{1} z_{1}^{2}, y=y_{1} z_{1}^{3}, z=z_{1}^{2}, w=w_{1} z_{1}^{3}$ and $D \cap \tilde{U}_{3}=\left\{z_{1}=0\right\} / \mathbb{Z}_{2}$.

In this chart, the pull-back of $\alpha$ by $E$ is given by

$$
E^{*} \alpha=z_{1}^{8}\left[\frac{9}{2}\left(x_{1}^{3} y_{1}+y_{1}^{3}\right) d z_{1}+\frac{3}{2} z_{1} x_{1}^{2} y_{1} d x_{1}+\frac{1}{2} z_{1}\left(x_{1}^{3}+3 y_{1}^{2}\right) d y_{1}+z_{1} \theta_{1}\right]
$$

where $\theta_{1}=E^{*} \alpha / z_{1}^{9}$. Therefore the foliation $\tilde{\mathcal{L}}_{\mathbb{C}}:=E^{*} \mathcal{L}_{\mathbb{C}}$ is defined by $\left.\alpha_{1}\right|_{\tilde{M}_{\mathbb{C}}}=0$, where

$$
\alpha_{1}=\frac{9}{2}\left(x_{1}^{3} y_{1}+y_{1}^{3}\right) d z_{1}+\frac{3}{2} z_{1} x_{1}^{2} y_{1} d x_{1}+\frac{1}{2} z_{1}\left(x_{1}^{3}+3 y_{1}^{2}\right) d y_{1}+z_{1} \theta_{1} .
$$

From (5.31) we have

$$
\tilde{C} \cap \tilde{U}_{3}=\left\{z_{1}=x_{1}^{3} y_{1}+y_{1}^{3}+w_{1}^{3}+w_{1}=0\right\} / \mathbb{Z}_{2}
$$

which implies that $\tilde{C}$ is invariant by $\tilde{\mathcal{L}}_{\mathbb{C}}$. Now it follows from (5.33) that the singular set of $\tilde{\mathcal{L}}_{\mathbb{C}}$ is given by

$$
\text { Sing } \tilde{\mathcal{L}}_{\mathbb{C}} \cap \tilde{U}_{3}=\left\{z_{1}=x_{1}^{3} y_{1}+y_{1}^{3}=w_{1}^{3}+w_{1}=0\right\} / \mathbb{Z}_{2} .
$$

In $\tilde{U}_{4}$ we introduce coordinates $\left(x_{2}, y_{2}, z_{2}, w_{2}\right)$ and $E$ has the expression

$$
E\left(x_{2}, y_{2}, z_{2}, w_{2}\right)=(x, y, z, w) \text {, }
$$

where $x=x_{2} w_{2}^{2}, y=y_{2} w_{2}^{3}, z=z_{2} w_{2}^{2}, w=w_{2}^{3}$ and $D \cap \tilde{U}_{4}=\left\{w_{2}=0\right\} / \mathbb{Z}_{3}$. 
In this chart, the foliation $\tilde{\mathcal{L}}_{\mathbb{C}}$ is defined by $\left.\alpha_{2}\right|_{\tilde{M}_{\mathbb{C}}}=0$, where

$$
\alpha_{2}=\frac{9}{2}\left(x_{2}^{3} y_{2}+y_{2}^{3}\right) d w_{2}+\frac{3}{2} w_{2} x_{2}^{2} y_{2} d x_{2}+\frac{1}{2} w_{2}\left(x_{2}^{3}+3 y_{2}^{2}\right) d y_{2}+w_{2} \beta_{1},
$$

and $\beta_{1}=E^{*} \theta / w_{2}^{9}$. Moreover

$$
\operatorname{Sing} \tilde{\mathcal{L}}_{\mathbb{C}} \cap \tilde{U}_{4}=\left\{w_{2}=x_{2}^{3} y_{2}+y_{2}^{3}=z_{2}^{3}+1=0\right\} / \mathbb{Z}_{3} .
$$

We claim that $\operatorname{Sing} D \subsetneq \operatorname{Sing} \tilde{\mathcal{L}}_{\mathbb{C}}$, where $D \cong \mathbb{P}(2,3,2,3)$ is the exceptional divisor of $E$. In fact, on $\tilde{U}_{3}$ the group acts via

$$
x_{1} \longmapsto x_{1}, \quad y_{1} \longmapsto-y_{1}, \quad w_{1} \longmapsto-w_{1}
$$

and on $\tilde{U}_{4}$ the group acts via

$$
x_{2} \longmapsto e^{4 \pi i / 3} x_{2}, \quad y_{2} \longmapsto y_{2}, \quad z_{2} \longmapsto e^{4 \pi i / 3} z_{2} .
$$

Therefore

$$
\operatorname{Sing} D \cap \tilde{U}_{3}=\left\{y_{1}=z_{1}=w_{1}=0\right\} / \mathbb{Z}_{2}
$$

and

$$
\text { Sing } D \cap \tilde{U}_{4}=\left\{x_{2}=z_{2}=w_{2}=0\right\} / \mathbb{Z}_{3},
$$

hence Sing $D \subsetneq \operatorname{Sing} \tilde{\mathcal{L}}_{\mathbb{C}}$, so the assertion is proved.

\subsubsection{End of the proof of case $E_{7}$}

Take $S=\tilde{C} \backslash \operatorname{Sing} \tilde{\mathcal{L}}_{\mathbb{C}}$, so that $S$ is a smooth leaf of $\tilde{\mathcal{L}}_{\mathbb{C}}$. Fix $q_{0} \in S$ and a transversal $\sum$ to $S$.

We work in the chart $\tilde{U}_{4}$. Take $q_{0}=(1,0,0,0)$ and the section $\sum=\{(1,0,0, t) \mid t \in$ $\mathbb{C}$ \}, parameterized by $t$. Call $G$ the holonomy group of the leaf $S$ of $\hat{\mathcal{L}}_{\mathbb{C}}$ in the section $\sum$. From (5.36), we have

$$
\operatorname{Sing} \tilde{\mathcal{L}}_{\mathbb{C}} \cap \tilde{U}_{4}=\left\{w_{2}=x_{2}^{3} y_{2}+y_{2}^{3}=z_{2}^{3}+1=0\right\} / \mathbb{Z}_{3} .
$$

The fundamental group $\pi_{1}\left(S, q_{0}\right)$ is generated by

$$
\pi_{1}\left(S, q_{0}\right)=\left\langle\gamma_{j}, \delta_{j}, \zeta_{j}: \delta_{j}^{3}=\zeta_{j}^{2}\right\rangle_{1 \leq j \leq 3}
$$

For each $j=1,2,3$, denote by $\rho_{j}$ a $3^{t d}$-primitive root of -1 , we have $\gamma_{j}$ are loops that turn around

$$
\left\{w_{2}=y_{2}=z_{2}-\rho_{j}=0\right\} \text { for all } 1 \leq j \leq 3
$$

and $\delta_{j}, \zeta_{j}$ are loops that turn around

$$
\left\{w_{2}=x_{2}^{3}+y_{2}^{2}=z_{2}-\rho_{j}=0\right\}, \text { for all } 1 \leq j \leq 3 .
$$

Therefore $G=\left\langle f_{j}, g_{j}, h_{j}\right\rangle_{1 \leq j \leq 3}$, where $f_{j}, g_{j}$ and $h_{j}$ correspond to $\left[\gamma_{j}\right],\left[\delta_{j}\right]$ and $\left[\zeta_{j}\right]$, respectively. We get from $(5.35)$ that $f_{j}^{\prime}(0)=e^{-2 \pi i / 9}, g_{j}^{\prime}(0)=e^{-2 \pi i / 3}$, $h_{j}^{\prime}(0)=e^{-\pi i}$, for all $1 \leq j \leq 3$. 


\subsection{Case $E_{8}$}

Write

$$
F(x, y)=\mathcal{R} e\left(x^{5}+y^{3}\right)+H(x, y, \bar{x}, \bar{y}) .
$$

The complexification $F_{\mathbb{C}}$ of $F$ can be written as

$$
F_{\mathbb{C}}(x, y, z, w)=\frac{1}{2}\left(x^{5}+y^{3}\right)+\frac{1}{2}\left(z^{5}+w^{3}\right)+H_{\mathbb{C}}(x, y, z, w),
$$

so that $M_{\mathbb{C}}=F_{\mathbb{C}}^{-1}(0) \subset\left(\mathbb{C}^{4}, 0\right)$. Note that $0 \in \mathbb{C}^{4}$ is an isolated singularity of $M_{\mathbb{C}}$ so the algebraic dimension of Sing $M$ is 0 .

Let us define the following algebraic hypersurface on $\mathbb{P}(3,5,3,5)$

$$
V_{M_{\mathbb{C}}}:=\left\{Z_{0}^{5}+Z_{1}^{3}+Z_{2}^{5}+Z_{3}^{3}=0\right\},
$$

where $\left[Z_{0}: Z_{1}: Z_{2}: Z_{3}\right] \in \mathbb{P}(3,5,3,5)$. Clearly Sing $M_{\mathbb{C}} \subset \operatorname{Sing} V_{M_{\mathbb{C}}}$ and $V_{M_{\mathbb{C}}}$ can be considered as a $V$-subvariety

$$
V_{M_{\mathbb{C}}} \subset Z=\mathbb{C}^{4} / \mathbb{Z}(3,5,3,5)
$$

Let $E: \tilde{Z} \rightarrow Z$ be the weighted blow-up with weight $\sigma=(3,5,3,5)$. Denote by $\tilde{M}_{\mathbb{C}}$ the strict transform of $M_{\mathbb{C}}$ by $E$. Take the exceptional divisor $D \cong \mathbb{P}_{\sigma}$ of $E$ with coordinates $\left(Z_{0}, Z_{1}, Z_{2}, Z_{3}\right) \in \mathbb{C}^{4} \backslash\{0\}$. The intersection of $\tilde{M}_{\mathbb{C}}$ with $\mathbb{P}_{\sigma}$ is the algebraic surface

$$
\tilde{C}=\tilde{M}_{\mathbb{C}} \cap \mathbb{P}_{\sigma}=\left\{Z_{0}^{5}+Z_{1}^{3}+Z_{2}^{5}+Z_{3}^{3}=0\right\}
$$

On the other hand, according to Remark (2.3), the foliation $\mathcal{L}_{\mathbb{C}}$ is defined by $\left.\alpha\right|_{M_{\mathbb{C}}^{*}}=$ 0 , where

$$
\alpha=\frac{5}{2} x^{4} d x+\frac{3}{2} y^{2} d y+\theta,
$$

where $\theta$ is a 1 -form with $j_{0}^{4}(\theta)=0$. For each $i=1, \ldots, 4$, we have the affine open sets of $E$ :

$$
\tilde{U}_{i}=\mathbb{C}^{4} / \mathbb{Z}_{a_{i}}\left(-a_{1}, \ldots, \quad{ }^{i-t h}, \ldots,-a_{4}\right),
$$

where $\sigma=\left(a_{1}, a_{2}, a_{3}, a_{4}\right)$. In $\tilde{U}_{3}$, the blow-up $E$ has the expression

$$
E\left(x_{1}, y_{1}, z_{1}, w_{1}\right)=(x, y, z, w)
$$

where $x=x_{1} z_{1}^{3}, y=y_{1} z_{1}^{5}, z=z_{1}^{3}, w=w_{1} z_{1}^{5}$ and $D \cap \tilde{U}_{3}=\left\{z_{1}=0\right\} / \mathbb{Z}_{3}$.

In this chart, the pull-back of $\alpha$ by $E$ is given by

$$
E^{*} \alpha=z_{1}^{14}\left[\frac{15}{2}\left(x_{1}^{5}+y_{1}^{3}\right) d z_{1}+\frac{5}{2} z_{1} x_{1}^{4} d x_{1}+\frac{3}{2} z_{1} y_{1}^{2} d y_{1}+z_{1} \theta_{1}\right]
$$


where $\theta_{1}=E^{*} \alpha / z_{1}^{15}$. Therefore the foliation $\tilde{\mathcal{L}}_{\mathbb{C}}=E^{*} \mathcal{L}_{\mathbb{C}}$ is defined by $\left.\alpha_{1}\right|_{\tilde{M}_{\mathbb{C}}}=$ 0 , where

$$
\alpha_{1}=\frac{15}{2}\left(x_{1}^{5}+y_{1}^{3}\right) d z_{1}+\frac{5}{2} z_{1} x_{1}^{4} d x_{1}+\frac{3}{2} z_{1} y_{1}^{2} d y_{1}+z_{1} \theta_{1} .
$$

From (5.38) we have

$$
\tilde{C} \cap \tilde{U}_{3}=\left\{z_{1}=x_{1}^{5}+y_{1}^{3}+w_{1}^{3}+1=0\right\} / \mathbb{Z}_{3}
$$

which implies that $\tilde{C}$ is invariant by $\tilde{\mathcal{L}}_{\mathbb{C}}$. From $(5.40)$, the singular set of $\tilde{\mathcal{L}}_{\mathbb{C}}$ is given by

$$
\operatorname{Sing} \tilde{\mathcal{L}}_{\mathbb{C}} \cap \tilde{U}_{3}=\left\{z_{1}=x_{1}^{5}+y_{1}^{3}=w_{1}^{3}+1=0\right\} / \mathbb{Z}_{3} .
$$

In $\tilde{U}_{4}$ we introduce coordinates $\left(x_{2}, y_{2}, z_{2}, w_{2}\right)$, and $E$ has the expression

$$
E\left(x_{2}, y_{2}, z_{2}, w_{2}\right)=(x, y, z, w),
$$

where $x=x_{2} w_{2}^{3}, y=y_{2} w_{2}^{5}, z=z_{2} w_{2}^{3}, w=w_{2}^{5}$ and $D \cap \tilde{U}_{4}=\left\{\bar{w}_{1}=0\right\} / \mathbb{Z}_{4}$.

In this chart, the foliation $\tilde{\mathcal{L}}_{\mathbb{C}}$ is defined by $\left.\alpha_{2}\right|_{\tilde{M}_{\mathbb{C}}}=0$, where

$$
\alpha_{2}=\frac{15}{2}\left(x_{2}^{5}+y_{2}^{3}\right) d w_{2}+\frac{5}{2} w_{2} x_{2}^{4} d x_{2}+\frac{3}{2} w_{2} y_{2}^{2} d y_{2}+w_{2} \beta_{1},
$$

and $\beta_{1}=E^{*} \theta / w_{2}^{15}$. Moreover

$$
\operatorname{Sing} \tilde{\mathcal{L}}_{\mathbb{C}} \cap \tilde{U}_{4}=\left\{w_{2}=x_{2}^{5}+y_{2}^{3}=z_{2}^{5}+1=0\right\} / \mathbb{Z}_{5}
$$

We assert that $\operatorname{Sing} D \cap \operatorname{Sing} \tilde{\mathcal{L}}_{\mathbb{C}}=\emptyset$, where $D \cong \mathbb{P}(3,5,3,5)$ is the exceptional divisor of $E$. In fact, on $D \cap \tilde{U}_{3}$ the group acts via

$$
x_{1} \longmapsto x_{1}, \quad y_{1} \longmapsto e^{10 \pi i / 3} y_{1}, \quad w_{1} \longmapsto e^{10 \pi i / 3} w_{1},
$$

and on $D \cap \tilde{U}_{4}$ the group acts via

$$
x_{2} \longmapsto e^{6 \pi i / 5} x_{2}, \quad y_{2} \longmapsto y_{2}, \quad z_{2} \longmapsto e^{6 \pi i / 5} z_{2} .
$$

Therefore

$$
\operatorname{Sing} D \cap \tilde{U}_{3}=\left\{y_{1}=z_{1}=w_{1}=0\right\} / \mathbb{Z}_{3}
$$

and

$$
\operatorname{Sing} D \cap \tilde{U}_{4}=\left\{x_{2}=z_{2}=w_{2}=0\right\} / \mathbb{Z}_{5},
$$

hence Sing $\tilde{\mathcal{L}}_{\mathbb{C}} \cap \operatorname{Sing} D=\emptyset$, so the assertion is proved. 


\subsubsection{End of the proof of case $E_{8}$}

Take $S=\tilde{C} \backslash \operatorname{Sing} \tilde{\mathcal{L}}_{\mathbb{C}}$, so that $S$ is a smooth leaf of $\tilde{\mathcal{L}}_{\mathbb{C}}$. Fix $q_{0} \in S \backslash \operatorname{Sing} D$ and a transversal $\sum$ to $S$.

We work in the chart $\tilde{U}_{3}$. Take $q_{0}=(1,0,0,0)$ and the section $\sum=\{(1,0, t, 0) \mid t \in$ $\mathbb{C}$ \}, parameterized by $t$. Call $G$ the holonomy group of the leaf $S$ of $\hat{\mathcal{L}}_{\mathbb{C}}$ in the section $\sum$. From (5.41), we have that

$$
\operatorname{Sing} \tilde{\mathcal{L}}_{\mathbb{C}} \cap \tilde{U}_{3}=\left\{z_{1}=x_{1}^{5}+y_{1}^{3}=w_{1}^{3}+1=0\right\} / \mathbb{Z}_{3}
$$

In this chart Sing $\tilde{\mathcal{L}}_{\mathbb{C}}$ has three irreducible components. For each $j=1,2,3$, denote by $\rho_{j}$ a $3^{t d}$-primitive root of -1 . The group $\pi_{1}\left(S, q_{0}\right)$ can be written is terms of generators and relations as

$$
\pi\left(S, q_{0}\right)=\left\langle\gamma_{j}, \zeta_{j}: \gamma_{j}^{3}=\zeta_{j}^{5}\right\rangle_{1 \leq j \leq 3}
$$

where $\gamma_{j}, \zeta_{j}$ are loops that turn around

$$
\left\{z_{1}=x_{1}^{5}+y_{1}^{3}=w_{1}-\rho_{j}=0\right\}, \text { for all } 1 \leq j \leq 3 .
$$

Therefore $G=\left\langle f_{j}, g_{j}\right\rangle_{1 \leq j \leq 3}$, where $f_{j}$ and $g_{j}$ correspond to $\left[\gamma_{j}\right]$ and $\left[\zeta_{j}\right]$, respectively. We get from (5.40) that $f_{j}^{\prime}(0)=e^{-2 \pi i / 3}, g_{j}^{\prime}(0)=e^{-2 \pi i / 5}$, for all $1 \leq j \leq 3$. This finishes the proof of Theorem 1 .

\section{References}

[1] V.I. ARNOLD, Normal forms of functions in the neighborhood of degenerate critical points, I, Russian Math. Surveys 29 (1974), 10-50.

[2] V. I. ARNOLD, Normal forms of functions near degenerate critical points, the Weyl groups $A_{k}, D_{k}, E_{k}$ and Lagrangian singularities, Functional Anal. Appl. 6 (1972), 254-272.

[3] M. S. Baouendi, P. Ebenfelt and L. P. Rothschild, "Real Submanifolds in Complex Space and their Mappings", Princeton Mathematical Series, 47, Princeton University Press, Princeton, NJ, 1999.

[4] E. BEDFORD, Holomorphic continuation of smooth functions over Levi-flat hypersurfaces, Trans. Amer. Math. Soc. 232 (1977), 323-341.

[5] D. BURns and X. Gong, Singular Levi-flat real analytic hypersurfaces, Amer. J. Math. 121 (1999), 23-53.

[6] M. BRunElla, Singular Levi-flat hypersurfaces and codimension-one foliations, Ann. Sc. Norm. Super. Pisa Cl. Sci. (5) 6 (2007), 661-672.

[7] M. BRUNELla Foliations on complex projective surfaces, In: "Dynamical Systems", Part II, Pubbl. Scuola Norm. Sup. Pisa (2003), 49-77.

[8] D. CERVEAU and A. LINS NeTO, Local Levi-flat hypersurfaces invariants by a codimension-one holomorphic foliation, Amer. J. Math. 133 (2011), 677-716.

[9] I. Dolgachev, Weighted projective varieties, In: "Group Actions and Vector Fields", J. B. Carrell (ed.), Lecture Notes in Mathematics, Vol. 956, Springer, Berlin, 1982, 34-71.

[10] A. H. DURFEE, Fifteen characterizations of rational double points and simple critical points, Enseign. Math. (2) 25 (1979), 131-163. 
[11] A. Fernández-PÉREZ, "Singular Levi-Flat Hypersurfaces. An Approach Through Holomorphic Foliations", Ph.D. Thesis, IMPA - Brazil, 2010.

[12] A. FERNÁNDEZ-PÉREZ, On normal forms of singular Levi-flat real analytic hypersurfaces, Bull. Braz. Math. Soc. (N.S.) (1) 42 (2011), 75-85.

[13] A. FERnÁndeZ-PÉREZ, On Levi-flat hypersurfaces tangent to holomorphic webs, Ann. Fac. Sci. Toulouse Math. (6) 20 (2011), 581-597.

[14] W. Fulton, "Introduction to Toric Varieties", Princeton Univ. Press, 1993.

[15] J. Kollar and S. Mori, Classification of three dimensional flips, J. Amer. Math. Soc. 5 (1992), 533-703.

[16] J. LEBL, Singular set of a Levi-flat hypersurface is Levi-flat, Math. Ann. 355 (2013), 11771199.

[17] F. LORAY, Pseudo-groupe d'une singularité de feuilletage holomorphe en dimension deux, Prépublication IRMAR (2005), ccsd-00016434.

[18] J. F. MATtEI and R. Moussu, Holonomie et intégrales premières, Ann. Éc. Norm. Supér. 13 (1980), 469-523.

[19] O.ZARISKI, On the topology of algebroid singularities, Amer. J. Math. 54 (1932), 455-465.

[20] H. ŻoŁADEK, "The Monodromy Group", Mathematics Institute of the Polish Academy of Sciences. Mathematical Monographs (New Series), 67, Birkhäuser Verlag, Basel, 2006.

Departamento de Matemática

Universidade Federal de Minas Gerais, UFMG

Av. Antônio Carlos, 6627 C.P. 702

31270-901 Belo Horizonte, MG Brazil

arturofp@mat.ufmg.br 